\title{
Deficiency in prohormone convertase PC1 impairs prohormone processing in Prader-Willi syndrome
}

\begin{abstract}
Lisa C. Burnett, ${ }^{1,2,3}$ Charles A. LeDuc, ${ }^{2,3,4}$ Carlos R. Sulsona, ${ }^{5}$ Daniel Paull, ${ }^{6}$ Richard Rausch, ${ }^{2,3}$ Sanaa Eddiry, ${ }^{7}$ Jayne F. Martin Carli, ${ }^{2,3,8}$ Michael V. Morabito, ${ }^{2,3}$ Alicja A. Skowronski, ${ }^{1,2,3}$ Gabriela Hubner, ${ }^{9}$ Matthew Zimmer, ${ }^{6}$ Liheng Wang, ${ }^{2,3}$ Robert Day, ${ }^{10}$ Brynn Levy, ${ }^{11}$ Ilene Fennoy, ${ }^{12}$ Beatrice Dubern, ${ }^{13}$ Christine Poitou, ${ }^{13}$ Karine Clement, ${ }^{13}$ Merlin G. Butler, ${ }^{14}$ Michael Rosenbaum, ${ }^{2,3}$ Jean Pierre Salles, ${ }^{7,15}$ Maithe Tauber, ${ }^{7,15,16}$ Daniel J. Driscoll,, ${ }^{5,17}$ Dieter Egli,, 2,3,6 and Rudolph L. Leibel $2,3,4$

${ }^{1}$ Institute of Human Nutrition, ${ }^{2}$ Department of Pediatrics, Division of Molecular Cenetics, and ${ }^{3}$ Naomi Berrie Diabetes Center, Columbia University, New York, New York, USA. ${ }^{4}$ New York Obesity Research Center, New York, New York, USA. 5Department of Pediatrics, Division of Cenetics and Metabolism, University of Florida College of Medicine Gainesville, Florida, USA. ${ }^{6}$ The New York Stem Cell Foundation Research Institute, New York, New York, USA. `Centre de Physiopathologie de Toulouse-Purpan, Université de Toulouse, CNRS UMR 5282, INSERM UMR 1043, Université Paul Sabatier, Toulouse, France. ${ }^{8}$ Department of Biochemistry and Molecular Biophysics, Columbia University, New York, New York, USA. ${ }^{9}$ Packer Collegiate Institute, New York, New York, USA. ${ }^{10}$ Institut de pharmacologie de Sherbrooke, Department of Surgery, Division of Urology, Faculty of Medicine and Health Sciences, Université de Sherbrooke, Sherbrooke, Quebec, Canada. "Department of Pathology and Cell Biology, Columbia University, New York, New York, USA. ${ }^{2}$ Department of Pediatrics, Division of Pediatric Diabetes, Endocrinology and Metabolism, Columbia University, New York, New York, USA. ${ }^{33}$ Institute of Cardiometabolism and Nutrition, Assistance Publique Hôpitaux de Paris, Sorbonne University, University Pierre et Marie-Curie, INSERM UMRS 1166, Paris, France. ${ }^{14}$ Department of Psychiatry and Behavioral Sciences, Division of Research and Genetics, Kansas University Medical Center, Kansas City, Kansas, USA. ${ }^{15}$ Unité d'Endocrinologie, Hôpital des Enfants, and ${ }^{16}$ Centre de Référence du Syndrome de PraderWilli, CHU Toulouse, Toulouse, France. ${ }^{17}$ Center for Epigenetics, University of Florida College of Medicine, Gainesville, Florida, USA.
\end{abstract}

Prader-Willi syndrome (PWS) is caused by a loss of paternally expressed genes in an imprinted region of chromosome 15q. Among the canonical PWS phenotypes are hyperphagic obesity, central hypogonadism, and low growth hormone (CH). Rare microdeletions in PWS patients define a 91-kb minimum critical deletion region encompassing 3 genes, including the noncoding RNA gene SNORD116. Here, we found that protein and transcript levels of nescient helix loop helix 2 (NHLH2) and the prohormone convertase PC1 (encoded by PCSK1) were reduced in PWS patient induced pluripotent stem cell-derived (iPSC-derived) neurons. Moreover, Nhlh2 and Pcsk1 expression were reduced in hypothalami of fasted Snord116 paternal knockout (Snord116 ${ }^{p-/ m+}$ ) mice. Hypothalamic Agrp and Npy remained elevated following refeeding in association with relative hyperphagia in Snord116 ${ }^{-/ m+}$ mice. Nhlh2-deficient mice display growth deficiencies as adolescents and hypogonadism, hyperphagia, and obesity as adults. Nhlh2 has also been shown to promote Pcsk1 expression. Humans and mice deficient in PC1 display hyperphagic obesity, hypogonadism, decreased $\mathrm{CH}$, and hypoinsulinemic diabetes due to impaired prohormone processing. Here, we found that Snord116 ${ }^{p-/ m+}$ mice displayed in vivo functional defects in prohormone processing of proinsulin, pro-GH-releasing hormone, and proghrelin in association with reductions in islet, hypothalamic, and stomach PC1 content. Our findings suggest that the major neuroendocrine features of PWS are due to PC1 deficiency.

\section{Introduction}

Prader-Willi syndrome (PWS) is the most common syndromic obesity, affecting 1 in 25,000 live births $(1,2)$. PWS results from a loss of paternally expressed genes at 15q11.2-q13 (Figure 1A) (3). Seventy percent of instances of PWS are due to a 5- to 6-Mb deletion in 15q11.2-q13 (Figure 1A). The major phenotypes of PWS include: hyperphagic obesity, hypogonadism, growth hormone (GH) deficiency, hyperghrelinemia, and relative hypoinsulinemia $(2,4)$. Five paternal microdeletion (118-237 kbp) PWS patients have been identified (5-9). The overlap among these patients' paternal deletion regions identifies a $91-\mathrm{kb}$ critical deletion region sufficient to cause the major physical and neuroendocrine pheno-

\section{Related Commentary: p. 98}

Conflict of interest: The authors have declared that no conflict of interest exists. Submitted: May 19, 2016; Accepted: October 20, 2016.

Reference information: J Clin Invest. 2017;127(1):293-305. doi:10.1172/JCI88648. types of PWS (Figure 1A). This region contains 3 noncoding RNA genes, including SNORD109A, SNORD116, and IPW. None of the extant PWS mouse models (more than a dozen have been generated) develop obesity (10). However, mice in which the paternal copy of Snord116 is deleted (Snord116 $6^{p-/ m+}$ ) display many of the neuroendocrine phenotypes of PWS, including hyperphagia, low $\mathrm{GH}$, decreased body length, impaired motor learning, hypoinsulinemia, and hyperghrelinemia $(11,12)$.

C/D box small nucleolar RNAs (snoRNAs) are noncoding small nucleolar RNAs that methylate ribosomal RNAs. However, there are no known ribosomal RNA targets for SNORD116-encoded snoRNAs (13). Thus, SNORD116 is thought to be a noncanonical snoRNA; and the mechanisms by which SNORD116 influences biological processes are unknown. Although the endocrine features and natural history of PWS have been well described, a molecular mechanism linking these features to the genes deleted in the PWS minimum critical deletion region has not been identified. Using mice in which the paternal copy of only Snord116 has 
A $\sim 500-720 \mathrm{~kb}$ $\sim 5.5 \mathrm{Mb}$

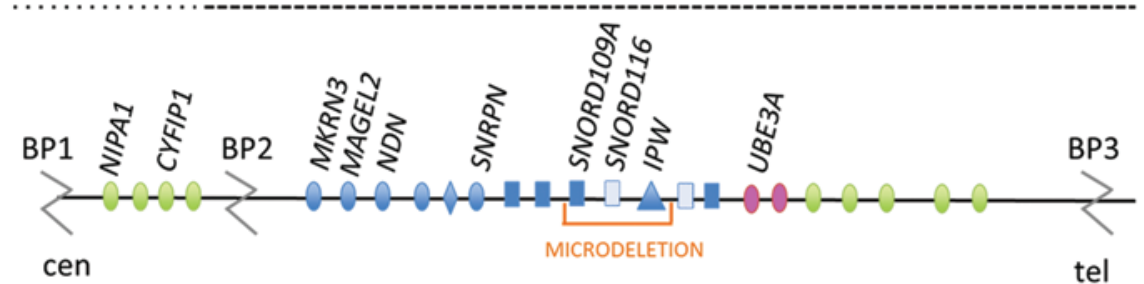

Type 1 deletion

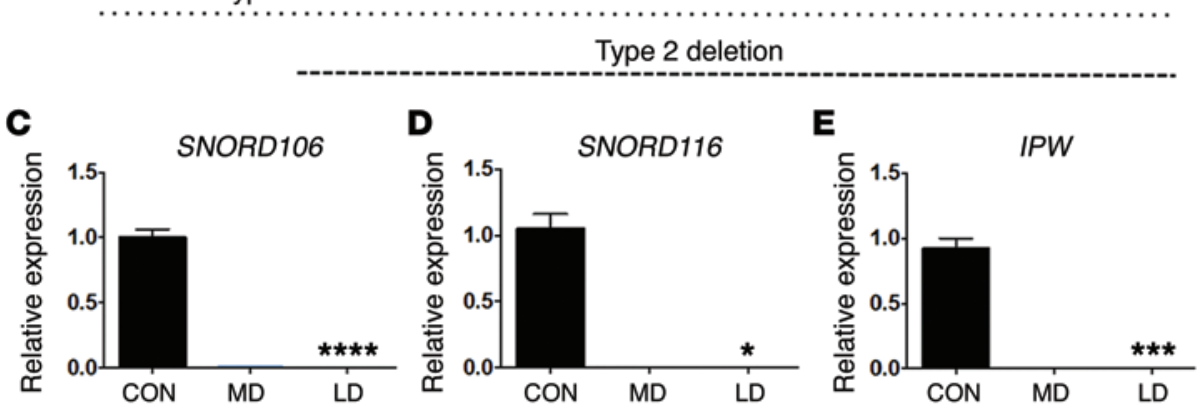

G

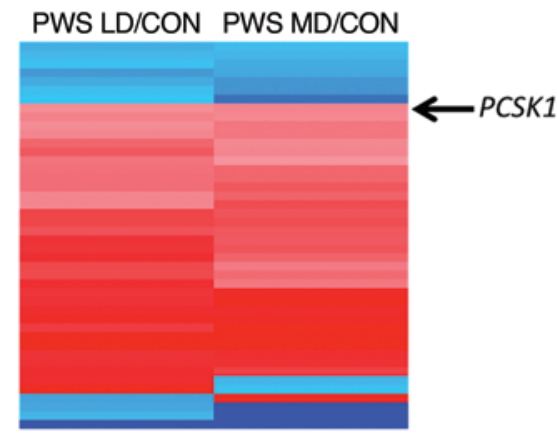

red=down, blue=up
H

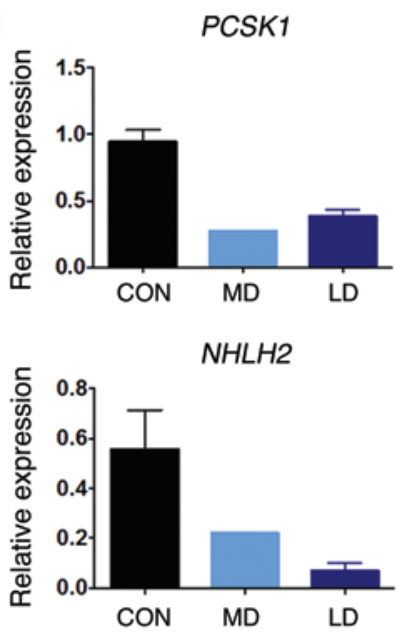

B
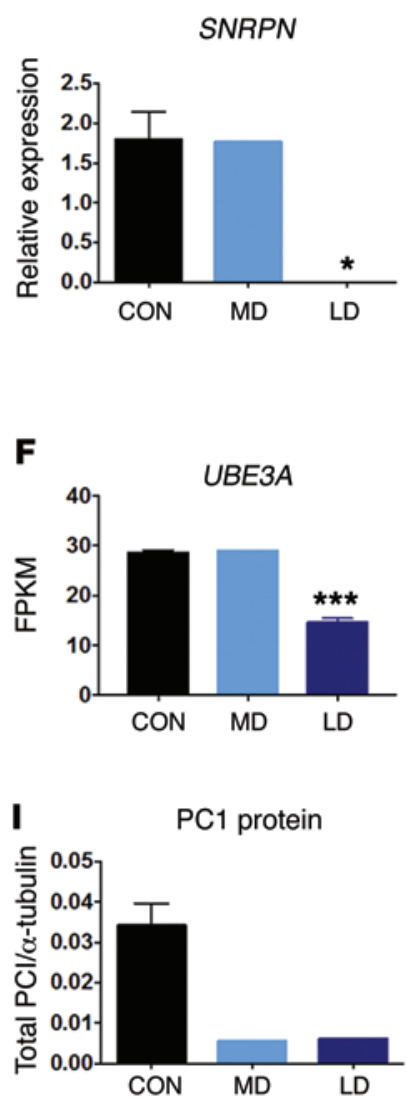

K NHLH2 protein
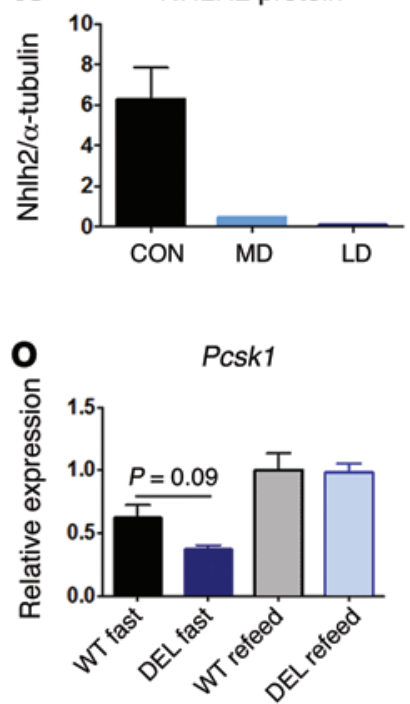
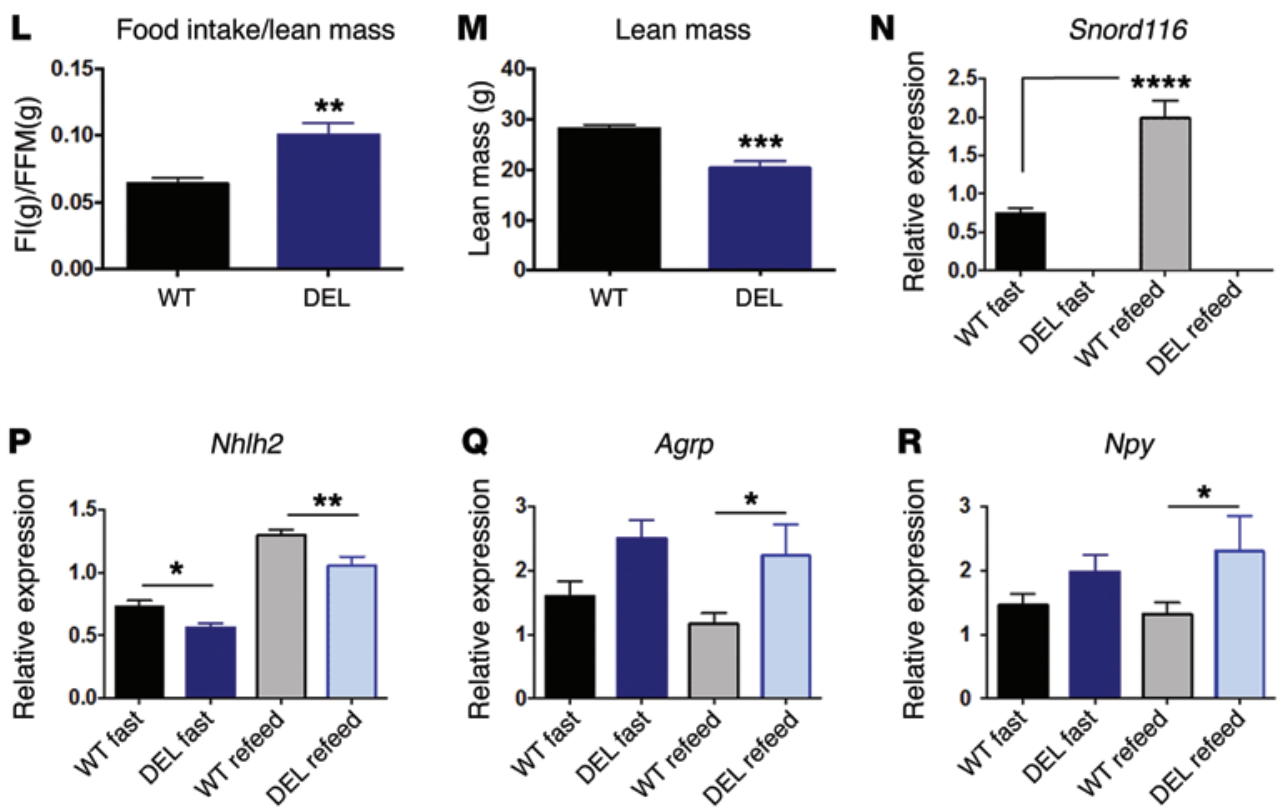
Figure 1. NHLH2 and PCSK1 are reduced in PWS iPSC-derived neurons and Snord116 ${ }^{-/ m+}$ (DEL) hypothalami. (A) Diagram of the PWS locus. Maternally expressed genes are shown in pink, paternally expressed genes in blue, non-imprinted genes in green. Protein-coding genes are shown as ovals, snoRNAs as rectangles, long noncoding RNAs as triangles, imprinting center as a diamond. Not drawn to scale. cen, centromere; tel, telomere. (B-F) Gene expression in the PWS locus following neuron differentiation ( $n=7$ control [CON], $n=1$ PWS MD [2 clones used], $n=3$ PWS LD). (C) RNA sequencing identified a downregulation in PCSK1 in PWS neurons $(n=7$ CON, $n=1$ PWS MD [2 clones used], $n=2$ PWS LD). This heatmap is also shown in Supplemental Figure 4A and includes the full list of all genes differentially expressed. (H and J) PCSK1 and NHLH2 gene expression levels from an independent differentiation experiment, as measured by qRT-PCR ( $n=7$ CON, $n=1$ PWS MD [2 clones used], $n=3$ PWS LD). (I and K) Quantification of PC1 and NHLH2 protein levels in iPSC-derived neurons ( $n=5$ CON [3 lines], $n=2$ PWS LD, $n=1$ PWS MD). (L and M) Food intake after 5 hours of refeeding ( $n=6 \mathrm{WT}, n=5 \mathrm{DEL}$ ). (N-R) Transcript levels in hypothalami at fasting and refeeding ( $n=11 \mathrm{WT}, n=13 \mathrm{DEL}$, overnight fasted; $n=15 \mathrm{WT}$, $n=14 \mathrm{DEL}$, 5-hour refed). All data are expressed as mean \pm SEM. B-F were analyzed with Kruskal-Wallis with post hoc Dunn's multiple comparison test; comparisons are against unaffected controls. $\mathbf{L}$ and $\mathbf{M}$ were analyzed with a 2-tailed, type 3 (assumes unequal variance) Student's $t$ test. N, $\mathbf{P}$, and $\mathbf{Q}$ were analyzed with 1-way ANOVA with Tukey's post hoc test. (0) WT fast and DEL fast were compared with a 2-tailed, type 3 Student's $t$ test. (R) WT refed and DEL refed were compared with a 2-tailed, type 3 Student's $t$ test. ${ }^{*} P<0.05,{ }^{* *} P<0.01,{ }^{* *} P<0.001,{ }^{* * *} P<0.0001$.

been deleted (Snord11 $\left.6^{p-m+}\right)$, induced pluripotent stem cell-derived (iPSC-derived) neurons from PWS patients, and plasma from PWS patients, we find that the major PWS clinical phenotypes can be accounted for by reduced expression of the prohormone processing enzyme prohormone convertase 1 (PC1, encoded by PCSK1).

\section{Results}

The major PWS phenotypes are likely of central nervous system origin (hyperphagia, central hypogonadism, GH deficiency, intellectual disability, developmental delay). We generated iPSC-derived neurons from 3 large deletion (LD) PWS patients and one microdeletion (MD) PWS patient with the smallest deletion (118$\mathrm{kb}$ deletion, chromosome 15q: 15:25,257,217-15:25,375,376) identified to date; iPSC-derived neurons were also differentiated from 6 unaffected individuals (Figure 1A and Supplemental Table 1; supplemental material available online with this article; doi:10.1172/ JCI88648DS1) (14). PWS iPSCs were differentiated to neurons using a modified dual SMAD signaling pathway inhibition protocol (15). PWS region genes were expressed in proportion to gene dosage in iPSC-derived neurons from unaffected controls, PWS LD patients, and a PWS microdeletion patient (Figure 1, B-F). PWS iPSC-derived neurons express canonical neural markers, including $\beta$-III-tubulin (TUJ1), NeuN, MAP2, and neural cell adhesion molecule (NCAM) (Supplemental Figure 1). PWS genotype status did not affect neuronal proliferation rates (percentage of Ki- $67^{+}$neurons) (Supplemental Figure 2) or the percentage of $\mathrm{NCAM}^{+}$neuron progenitors (measured by FACS) at day 12 or day 34 of differentiation (Supplemental Figure 3). These data suggest that general neuron differentiation efficiency using the modified dual SMAD inhibition protocol is similar between unaffected control and PWS large and microdeletion iPSC lines. These data further suggest that transcriptional differences between genotypes are not due solely to gross defects in neuronal differentiation in PWS lines.
Unidirectional RNA sequencing of iPSC/hESC-derived neurons FACS-sorted for NCAM (Supplemental Figure 4) identified PCSK1 among the top downregulated genes (Figure 1G). Pathway analysis using DAVID (https://david.ncifcrf.gov/) found that most dysregulated pathways included PCSK1: for example, "signal peptide," "response to steroid hormone stimulus," "pituitary gland development," "pancreas development," and "diencephalon development" (Supplemental Table 5). Quantitative RT-PCR (qRT-PCR) from an independent differentiation experiment confirmed the downregulation of PC1 in PWS iPSC-derived neurons (Figure 1H). By Western blotting, $\mathrm{PC} 1$ protein is reduced $>80 \%$ in both microdeletion and LD PWS iPSC-derived neurons (Figure 1I and Supplemental Figure 5). While both PCSK1 and nescient helix loop helix 2 (NHLH2) were among the top downregulated genes in PWS iPSC-derived neurons in a pilot RNA sequencing study, downregulation of $N H L H 2$ was not statistically significant $(P=$ 0.52 ) in the follow-up RNA sequencing study. However, qRT-PCR and Western blotting showed downregulation of NHLH2 at both the transcript and protein levels in PWS microdeletion and LD iPSC-derived neurons compared with unaffected controls (Figure 1, J and K, and Supplemental Figure 6). Because of the known relationship between Nhlh2 and Pcsk1, we thought it important to report the Nhlh2 data.

Nhlh2-null mice are obese, hypogonadal, and display reduced linear growth (16). Hypothalamic levels of Pcsk1 transcript and PC1 protein are reduced by more than $50 \%$ in these animals (17). NHLH2 transcript levels were reduced 1.5-fold in lymphoblast RNA from individuals with PWS compared with controls (18). NHLH2 is a basic helix-loop-helix transcription factor that positively regulates PCSK1 transcription as a heterodimer with STAT3 (19). Nhlh2 binds to E-box motifs within the PCSK1 promoter that are adjacent to STAT3 binding sites (19).

$\mathrm{PC} 1$ is the protein product of the PCSK1 gene. PC1 is an enzyme involved in the posttranslational modification of propeptides. PC1 is most active in the acidic environment of secretory vesicles, cleaving proproteins at dibasic residues (20). In conjunction with other prohormone convertases, including PC2, carboxypeptidase $\mathrm{E}$ (CPE), and furin, PC1 participates in the excision the hormone's pro-domain, generally increasing the hormone's bioactivity (21). Known substrates of PC1 include pro-opiomelanocortin (POMC), pro-gonadotropin-releasing hormone (proGnRH), pro-GH-releasing hormone (proGHRH), proinsulin, and proghrelin. Individuals hypomorphic for PCSK1 are hyperphagic, obese, hypogonadal, have low circulating GH levels, and have hyperproinsulinemia associated with hypoinsulinemia (22). Nhlh2-null and Pcsk1 $1^{\text {N222D }}$ mice develop obesity that is associated with impaired hypothalamic processing of POMC to $\alpha$-MSH as well as impaired proprotein processing of other hormones $(17,23)$. In humans and mice, hypomorphic mutations of POMC or the $\alpha$-MSH receptor cause obesity $(24,25)$. Nhlh2- and Pcsk1-null mice show reduced hypothalamic processing of pro-thyrotropin-releasing hormone (proTRH) to TRH $(17,26)$.

SNORD116 may act upstream of NHLH2 and/or PCSK1. We sought to determine whether we could detect downregulation of Nhlh2 and Pcsk1 in vivo in mice in which only the paternal allele of Snord116 (Snord116 ${ }^{p-m+}$ ) is deleted (11). Snord116 is not expressed in hypothalami of Snord116 $6^{p-m+}$ mice (Figure 1N). In WT animals, 
A
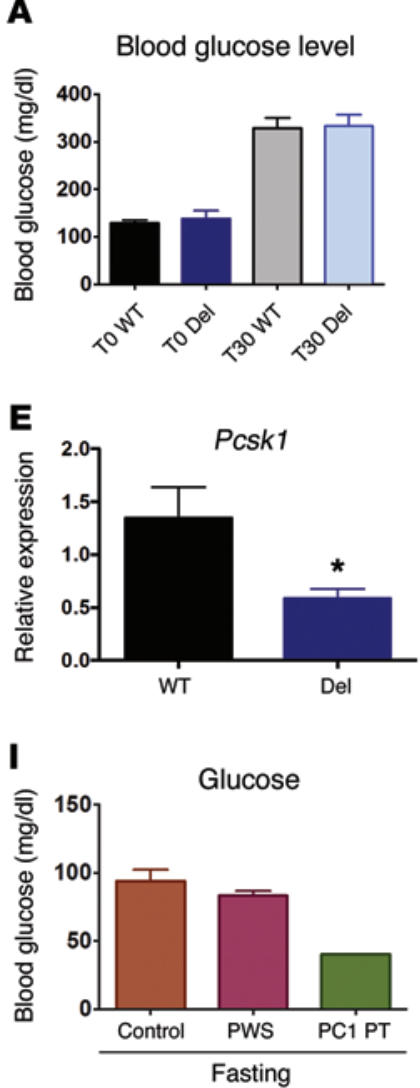

B
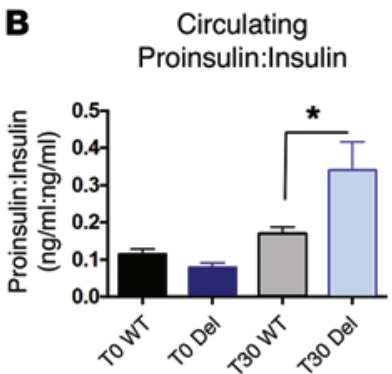

$\mathbf{F}$

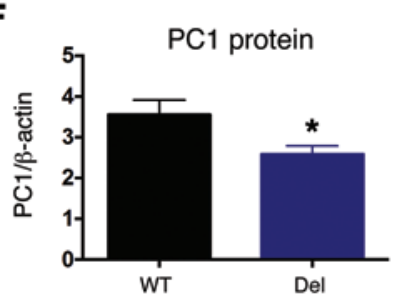

J

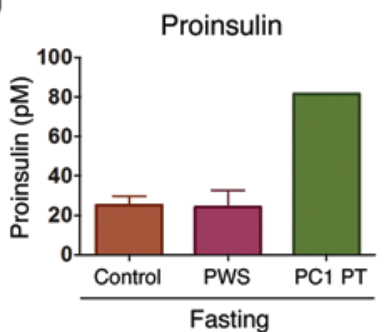

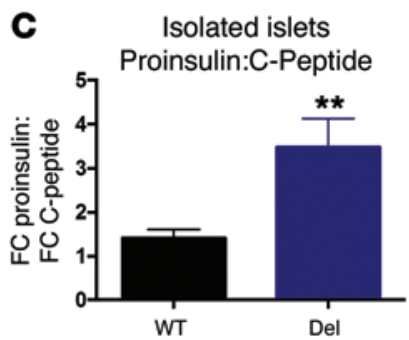

D

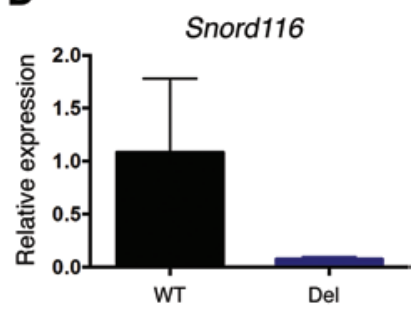

G

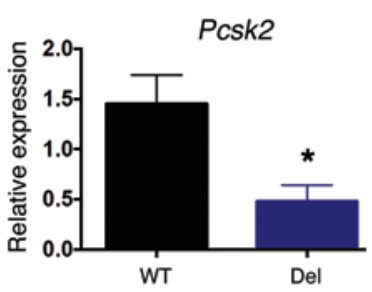

H

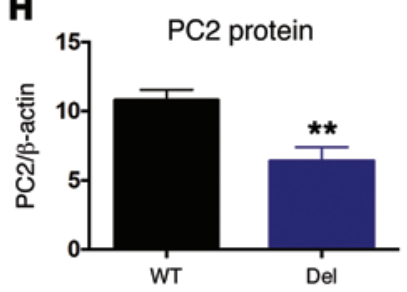

$\mathbf{L}$

$\mathbf{K}$

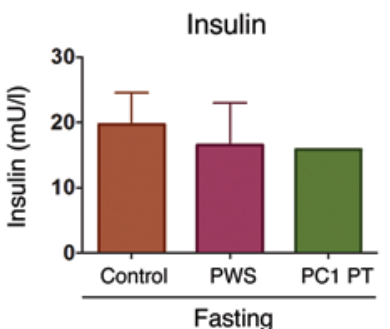

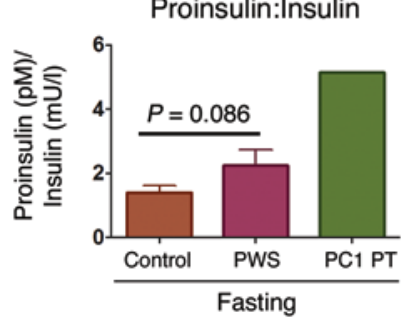

Figure 2. Proinsulin processing is impaired in Snord116 ${ }^{p / m+}$ (DEL) mice, and human PWS patients have impaired proinsulin processing compared with age- and BMI-matched controls. (A) There is no difference in blood glucose levels by genotype ( $n=9 \mathrm{WT}, n=7 \mathrm{DEL}$ ). (B) An increase in the ratio of proinsulin to insulin is detected 30 minutes after glucose stimulation ( $n=9 \mathrm{WT}, n=7 \mathrm{DEL}$ ). (C) The ratio of proinsulin to C-peptide in culture media is also increased in Snord116 ${ }^{p-/ m+}$ isolated islets ( $\left.n=9 \mathrm{WT}, n=10 \mathrm{DEL}\right)$. (D) Snord116 is not expressed in Snord116 ${ }^{p-/ m+}$ islets $(n=6 \mathrm{WT}, n=5 \mathrm{DEL})$. (E and G) Pcsk1 and Pcsk2 are downregulated in Snord116 $6^{-/ m+}$ islets $(n=6 \mathrm{WT}, n=5 \mathrm{DEL})$. ( $\mathbf{F}$ and $\left.\mathbf{H}\right)$ Both PC1 and PC2 protein levels are decreased in Snord116 $6^{p-/ m+}$ islets ( $n=15 \mathrm{WT}, n=15 \mathrm{DEL}$ ). (I) Fasting plasma glucose levels of PWS patients, a patient with an inactivating mutation in PCSK1, and age- and BMI-matched controls were measured ( $n=25$ unaffected controls, $n=16$ PWS patients, $n=1$ PCSK1 mutation patient). (J and $\mathbf{K}$ ) Fasting proinsulin and insulin levels were measured ( $n=25$ unaffected controls, $n=16$ PWS patients, $n=1$ PCSK1 mutation patient). (L) The plasma proinsulin to insulin ratio is increased by $60 \%$ in individuals with PWS at fasting compared with control $(P=0.086)(n=25$ age/BMI-matched controls, $n=16$ PWS patients, $n=1$ PCSK1 mutation patient). This effect is intermediate to that observed in the patients segregating for hypomorphic alleles of $P C S K 1$ and manifesting a $170 \%$ increase in plasma proinsulin to insulin ratio. All data are expressed as mean \pm SEM. A and $\mathbf{B}$ were analyzed with 1-way ANOVA with post hoc Tukey test; comparisons between all groups. C-H were analyzed with a 2-tailed, type 3 Student's $t$ test. Comparison between CON and PWS in I-L was done with a 2-tailed, type 3 Student's $t$ test; there is no statistical analysis included the PC1 mutation patient, as there was only data from 1 patient. ${ }^{*} P<0.05$, ${ }^{* *} P<0.01$ vs. WT.

Snord116 transcript levels increase $171 \%$ following 5-hour refeeding, suggesting that Snord116 may have anorexigenic properties (Figure $1 \mathrm{~N})$. In agreement with a putative anorexigenic function for Snord116, the food intake during 5-hour refeeding of Snor$d 116^{p-/ m+}$ mice is $67 \%$ greater than that of WT littermates (Figure $1, \mathrm{~L}$ and M). Levels of Pcsk1 transcripts are reduced $41 \%$ after fasting but are unchanged after refeeding (Figure 1O). Compared with WT, in Snord116 $6^{p-m+}$ mice hypothalamic Nhlh 2 transcript levels are decreased at fasting (-23\%) and refeeding (-19\%) (Figure 1P).

Snord $116^{p-/ m+}$ mice ingest more calories than WT littermates relative to lean mass during 5 -hour refeeding (Figure 1L). Furthermore, despite their reduced total body weights, accounted for by reductions in both lean and fat mass, Snord116 $6^{p-/ m+}$ mice consumed the same number of calories as WT littermates over the course of 6 days in calorimetry (Supplemental Figure 7, A, G, and H, and see below). When daily food intake was normalized to body weight, the results indicated that Snord $116^{p-m+}$ mice are actually hyperphagic relative to body weight (Supplemental Figure 7B). With energy expenditure plotted against $\mathrm{BW}^{2 / 3}$, we were able to use a single linear regression equation to describe both Snord116 $6^{p-/ m+}$ and WT data sets; this suggests that the increased energy expenditure of Snord11 $6^{p-/ m+}$ mice may be accounted for by increased heat loss due to higher surface area to body mass ratio of Snord $116^{p-/ m+}$ animals (Supplemental Figure 7C). No effects on 24-hour respiratory exchange ratio or mean daily movement were detected (Supplemental Figure 7, D and E).

Consistent with the relative hyperphagia of $\operatorname{Snord} 116^{p-/ m+}$ mice, hypothalamic transcript levels of the orexigenic neuropeptides neuropeptide Y $(N p y)$ and agouti-related peptide (Agrp) were elevated in Snord116 $6^{-/ m+}$ animals (Figure 1, L, Q, and R, and Supplemental Figure 7). AgRP is also processed by PC1, and $\mathrm{Pcsk1}^{-/-}$mice have 3.3-fold more full-length AgRP in the hypothal- 
A

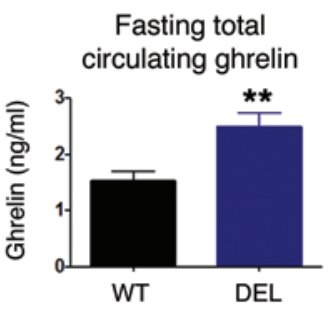

E

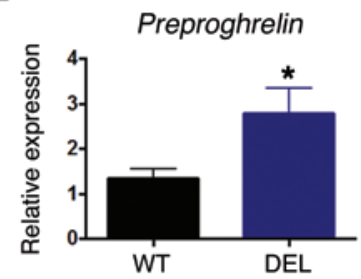

I

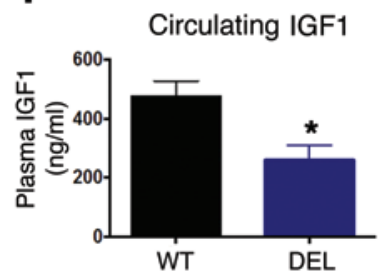

B

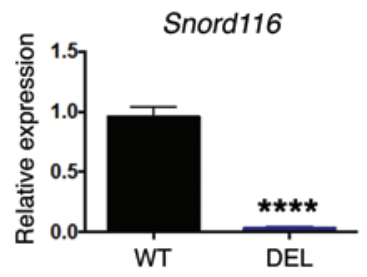

$\mathbf{F}$

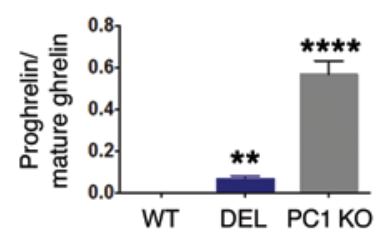

J

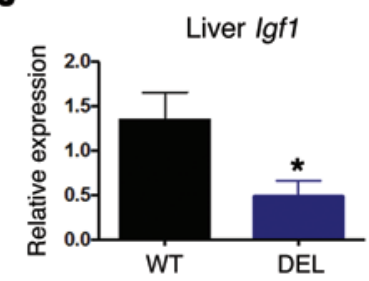

C

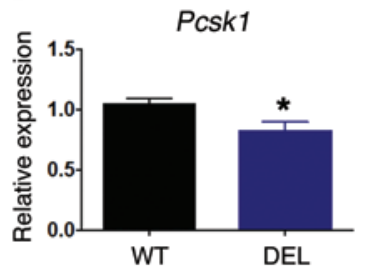

G

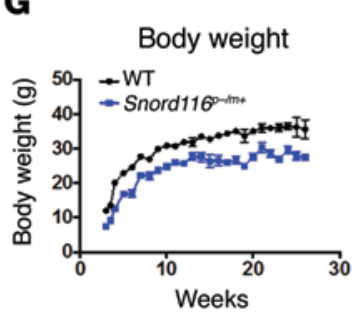

K

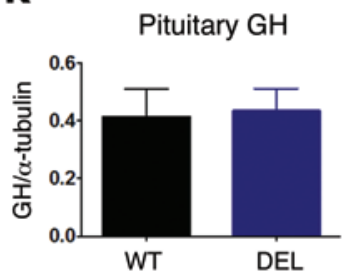

D

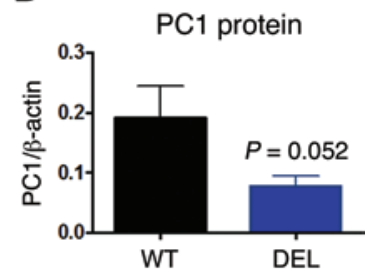

H

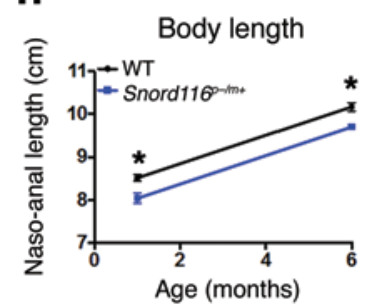

L Hypothalamic proGHRH/GHRH

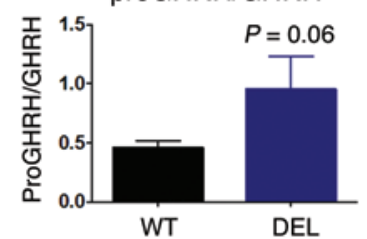

Figure 3. Proghrelin and ProGHRH processing are impaired in Snord116 ${ }^{p-/ m+}$ mice. (A) Levels of total circulating ghrelin are increased in Snord116 $6^{p / m+}$ mice $(n=21$ WT, $n=17 \mathrm{DEL})$. (B) Snord116 is expressed in stomachs of WT mice but not of Snord116 ${ }^{p-/ m+}$ mice $(n=6 \mathrm{WT}, n=6 \mathrm{DEL})$. (C) Pcsk1 transcript and levels are decreased in stomachs of Snord116 $6^{p / m+}$ mice compared with WT littermates ( $n=6 \mathrm{WT}, n=6 \mathrm{DEL}$ ). (D) PC1 protein is decreased in the stomach of Snord116 ${ }^{p-/ m+}$ mice, $p=0.052$ ( $n=6 \mathrm{WT}, n=6 \mathrm{DEL})$. (E) Preproghrelin transcript (Ghrl) is increased in Snord116 ${ }^{-/ / m+}$ mice $(n=6 \mathrm{WT}, n=6 \mathrm{DEL})$. (F) The ratio of proghrelin to ghrelin is increased in the stomachs of Snord116 $6^{-/ m+}$ and Psck1 $1^{--}$(PC1-KO) animals ( $n=9$ WT; $n=7$ DEL; $n=2$ PC1 KO). (G and H) From weaning throughout adulthood, Snord116 ${ }^{p-/ m+}$ mice weigh less and are shorter than WT littermates (G: $n=4-20$ mice/time point; $\mathbf{H : ~} n=6 \mathrm{WT}, n=7 \mathrm{DEL}$ ). (I) Circulating IGF1 is reduced by $45 \%$ in P30 Snord116 ${ }^{p-/ m+}$ mice compared with WT littermates ( $n=6 \mathrm{WT}, n=7 \mathrm{DEL}$ ). (J) Transcript levels of liver lgf1 are reduced by 64\% in Snord116 $6^{p / m+}$ P30 livers compared with WT littermates ( $n=6 \mathrm{WT}, n=7 \mathrm{DEL})$. (K) There is no change in pituitary $\mathrm{CH}$ content between Snord11 $6^{p-/ m+}$ mice and WT ( $\left.n=5 \mathrm{WT}, n=4 \mathrm{DEL}\right)$. (L) An increased ratio of hypothalamic proGHRH to GHRH content suggests that proGHRH to GHRH processing is impaired in Snord116 $6^{p / m+}$ animals compared with WT littermates $(n=14$ $\mathrm{WT}, n=11 \mathrm{DEL}$ ). All data are expressed as mean \pm SEM. A-E and $\mathbf{G}-\mathbf{L}$ were analyzed with a 2-tailed, type 3 Student's $t$ test. F was analyzed with 1 -way ANOVA with post hoc Tukey test; comparisons between all groups. ${ }^{*} P<0.05,{ }^{* *} P<0.01,{ }^{* * *} P<0.0001 \mathrm{vs}$. WT.

amus; however, unlike POMC, processing is not necessary for antagonist activity of AgRP at the melanocortin receptor MC4R $(27,28) . P c s k 1^{-/-}$and Snord $116^{p-/ m+}$ mice are runted, confounding effects of genotype on obesity-related phenotypes. Nonetheless, Snord116 $6^{p-m+}$ mice also display long- and short-term increased energy intake relative to their diminished lean mass, associated with decreased hypothalamic Nhlh2 and Pcsk1 at fasting and persistence of increased hypothalamic Npy and Agrp following feeding (Figure 1, L-R, and Supplemental Figure 7).

Based on the data in Figure 1, we hypothesized that specific endocrine phenotypes of PWS might be a consequence of defective prohormone processing owing to a functional deficiency of PC1 activity. We tested this hypothesis by measuring the processing of proinsulin to insulin, proGHRH to GHRH, and proghrelin to ghrelin in vivo in Snord11 $6^{p-/ m+}$ mice and WT littermates, as well as in human PWS patients and controls matched for age and BMI (Figures 2, 3, and 4).

Proinsulin is processed to insulin and C-peptide in $\beta$ cells by the combined actions of PC1 and PC2 (29). Patients with PCSK1-inactivating mutations display hyperproinsulinemia and low circulating concentrations of insulin $(30,31)$. Snord11 $6^{p-/ m+}$ and WT mice were fasted overnight and injected intraperitoneally with $3 \mathrm{mg}$ glucose per kg body weight. Fasting and post-injection blood glucose levels did not differ by genotype (Figure 2A). At 30 minutes, Snord116 $6^{p-m+}$ mice displayed elevated plasma proinsulin/ insulin ratios (Figure 2B and Supplemental Figure 8). Isolated islets of Snord116 ${ }^{p-m+}$ mice secreted higher ratios of proinsulin:c-peptide in response to $20 \mathrm{mM}$ glucose (Figure 2C). While Snord116 expression is highest in the brain, qRT-PCR in WT mice detects Snord116 expression in most tissues, including isolated islets, the pituitary, adrenal gland, stomach, small intestine, and testes (Supplemental Figure 9). Snord116 is expressed in WT isolated islets but not those from Snord116 $6^{p-m+}$ mice (Figure 2D). Pcsk1 and Pcsk2 transcripts and PC1 and PC2 protein levels were decreased in islets from Snord11 $6^{p-m+}$ mice (Figure 2, E-H, and Supplemental Figures 10 and 11). These results suggest that loss of Snord116 alone is sufficient to cause a downregulation in $\mathrm{PC} 1$ and $\mathrm{PC} 2$, with a resulting functional impairment in proinsulin processing in vivo.

We identified a $60 \%$ increase in the ratio of proinsulin to insulin in fasting plasma of individuals with PWS as compared with 


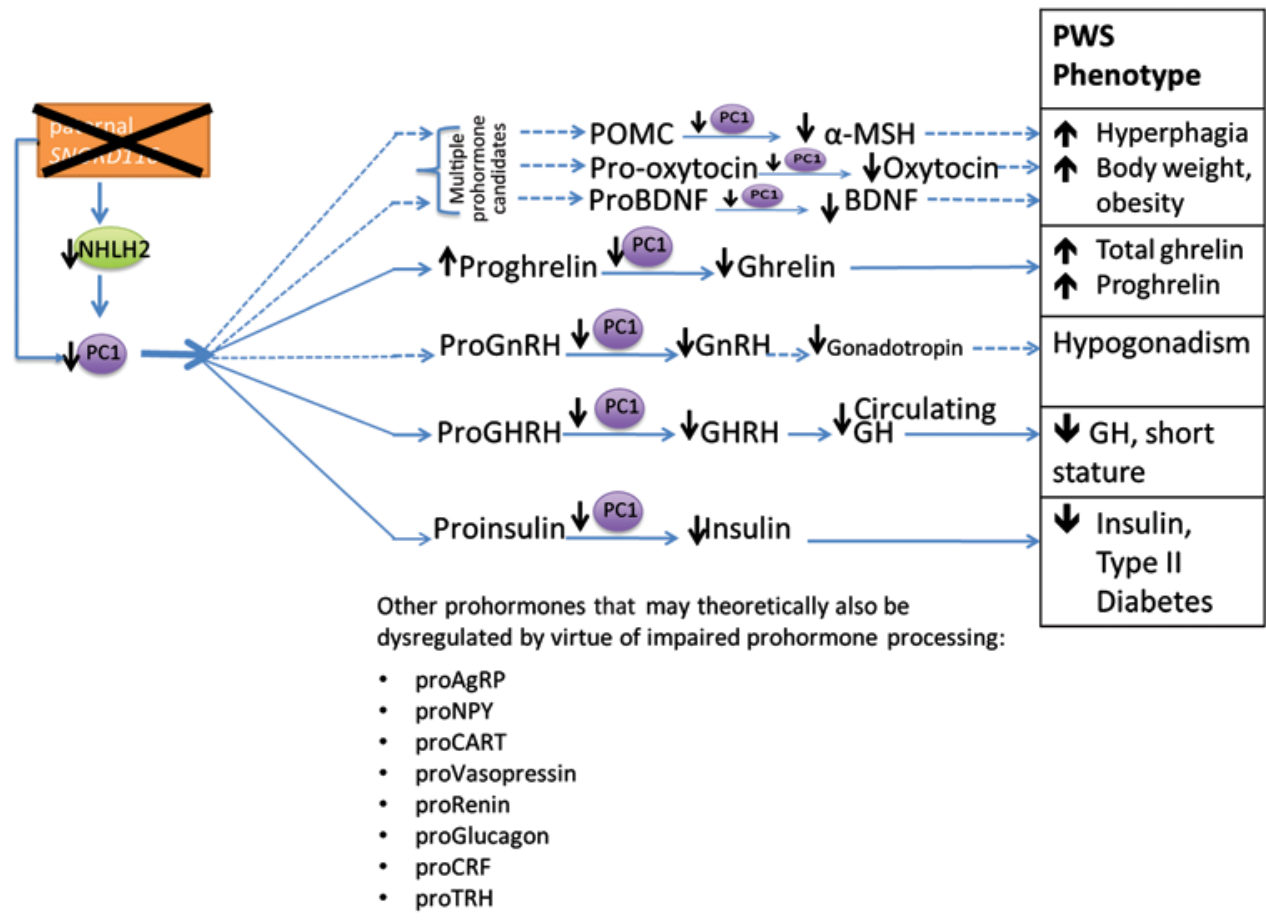

Figure 4. Deficiencies in PC1 drive the major neuroendocrine phenotypes of PWS. This schematic illustrates our hypothesis that paternal loss of SNORD116 is sufficient to cause deficiencies in the expression of NHLH2 and PCSK1 (PC1), resulting in impaired prohormone processing. We propose that deficiencies in prohormone processing (owing to deficits in PC1 production) explain the major neuroendocrine phenotypes of PWS.

$>10$-fold in stomachs of $\mathrm{Pcsk1}^{+/}$ mice, suggesting a possible negative feedback loop (Supplemental Figure 13). Ratios of proghrelin to mature ghrelin protein are elevated in Snord116 $6^{p-/ m+}$ stomach lysates (Figure 3F and Supplemental Figure 14). Stomach lysates from PC1-null mice were included as a positive control for impaired proghrelin processing. The processing defect in Snord $116^{p-/ m+}$ mice appears to be less severe than that of Pc1-null mice, consistent with the $\sim 50 \%$ decrease in PC1 protein in their stomachs. The antibodies used in conventional ghrelin assays to detect circulating total ghrelin in mouse and human also detect proghrelin (Supplemental Figure 15). Specifically, multiple groups have identified elevated circulating total ghrelin in individuals with PWS using the Phoenix Peptide RK-031-30 RIA kit; Supplemental Figure 15 shows that this antibody detects $14-\mathrm{kDa}$ and $17-\mathrm{kDa}$ proghrelin species in addition to 3.4-kDa mature ghrelin

age- and BMI-matched controls $(P=0.08)$ (Figure 2L and Supplemental Table 2). There was no difference in blood glucose or proinsulin concentrations between the subjects. The increase in the proinsulin/insulin ratio was driven by reductions in mature insulin (Figure 2, I-L). Plasma from a fasted patient compound heterozygous for inactivating mutations (heterozygous deletion c.71del/p. Ser24Met ${ }^{*} 73 /$ stop codon position 72 ) in PCSK1 was included as a control. The ratio of proinsulin to insulin was increased $267 \%$ compared with that in unaffected controls (Figure $2 \mathrm{~K}$ ). As we hypothesize that individuals with PWS have a downregulation of PC1, not a complete loss of function, an intermediate impairment in proinsulin processing as compared with PCSK1 mutation patients is expected.

Ghrelin (encoded by $G h r l$ ) is an orexigenic peptide produced in the stomach that is an endogenous ligand for the GH secretagogue receptor (GHSR) (32). Hyperghrelinemia preceding the onset of obesity is a clinical feature of PWS that is not seen in common obesity or other syndromic or monogenetic obesities (33-39). Fasted Snord116 $6^{p-/ m+}$ mice displayed increased levels of circulating total ghrelin (Figure 3, A and B). Pcsk1 transcript levels were 20\% lower in the stomachs of Snord116 $6^{p-m+}$ animals (Figure 3C). A trend toward decreased PC1 protein was identified in stomach lysates from Snor$d 116^{p-/ m+}$ mice compared with WT littermates $(P=0.052)$ (Figure 3D and Supplemental Figure 12). Transcript levels of Ghrl, which encodes the full-length preproghrelin protein, are reported to be elevated by $40 \%$ in the stomachs of PC1-null mice (40). This is the only other rodent obesity model in which hyperghrelinemia has been identified. Ghrl transcript was also increased in the stomachs of the Snord116 $6^{p-m+}$ mice (Figure 3E). Snord116 transcripts are increased
(33, 41-44). Thus, the reported hyperghrelinemia in PWS patients as well as the Snord116 ${ }^{p-/ m+}$ mice likely reflects elevated circulating proghrelin, consistent with our results in Snord116 $\mathrm{p}^{-/ m+}$ stomach lysates (Figure 3, A-F, and Supplemental Figures 14 and 15).

ProGHRH is processed to its active form by PC1 in the arcuate nucleus of the hypothalamus $(45,46)$. Similar to PWS patients, PC1-null mice, and PC1-deficient patients, Snord116 $6^{p-m+}$ mice exhibit growth retardation and decreased circulating GH/IGF1 levels (3, 46-48). At weaning and throughout adulthood, Snor$d 116^{p-/ m+}$ animals display decreased body length and weight (Figure 3, G and H). GH stimulates IGF1 production in the liver, generating the major endocrine mediator of $\mathrm{GH}$ effects on somatic growth. Both circulating IGF1 and liver transcript levels of IgfI were decreased in Snord116 ${ }^{p-/ m+}$ mice (Figure 3, I and J).

Although circulating levels of IGF1 and liver transcript levels of Igf 1 were decreased, GH levels in the pituitaries of four-week old, overnight fasted male Snord116 $6^{p-/ m+}$ mice were the same as WT littermates, suggesting impaired release of GH rather than impaired pituitary production of GH (Figure 3K and Supplemental Figure 16). Mice null for Nhlh2 have reduced growth from 4 to 7 weeks of age and a $>50 \%$ reduction in hypothalamic PC1 $(16,17)$. In PC1-null animals, impaired release of GH is associated with a defect in the processing of proGHRH to GHRH (46). We detected a trend toward an elevated ratio of proGHRH to GHRH in hypothalamic lysates of 6-week-old overnight-fasted Snord116 $6^{\mathrm{p}-/ m+}$ mice as compared with their WT littermates (Figure 3L). Nhlh2 and Pcsk1 transcripts are reduced in the hypothalami of fasted Snord116 $6^{p-m+}$ mice (Figure 1, O and P). Individuals with PWS do respond to GHRH administration by release of $\mathrm{GH}$ into the plasma 
(49). This finding is consistent with the inference that the short stature and decreased GH levels of individuals with PWS results from a hypothalamic deficiency of PC1.

\section{Discussion}

Our finding of reductions in NHLH2 and PC1 at both the transcript and protein levels in PWS iPSC-derived neurons is consistent with the possibility that the major neuroendocrine phenotypes of PWS are due to defects in prohormone processing. The deleted region of the PWS microdeletion patient studied here includes only 3 noncoding RNA genes: SNORD109A, SNORD116, and IPW. Mice lacking just the paternal copy of Snord116 have reduced levels of hypothalamic Nhlh 2 and Pcsk1 during fasting. Snord116 $6^{p-/ m+}$ mice display functional defects in prohormone processing in vivo: the processing of proinsulin, proGHRH, and proghrelin was impaired in these animals associated with tissue-specific reductions in PC1. The impaired processing of proGHRH was associated with the physiological readout of decreased circulating IGF1 and the anatomical phenotype of runted Snord116 $6^{p-m+}$ mice that have reduced body weight and body length. Furthermore, impaired processing of proghrelin to ghrelin was associated with increased circulating total ghrelin during fasting in Snord $116^{p-/ m+}$ mice. Finally, an elevated ratio of proinsulin to insulin during fasting was detected in the plasma of PWS patients compared with age- and BMI-matched controls, suggesting that proinsulin processing is impaired in human PWS patients as well.

These data led to the formulation of the hypothesis, presented graphically in Figure 4, that PC1 deficiency due to paternal deletion of SNORD116 is the major driver of the salient clinical phenotypes of the PWS. The major phenotypes of PWS that could be accounted for by PC1 deficiency include hyperphagic obesity (multiple candidate prohormones), low GH and short stature (impaired proGHRH processing), hypogonadism (impaired proGnRH processing), hyperghrelinemia (impaired proghrelin processing), relative hypoinsulinemia and type 2 diabetes mellitus (impaired proinsulin processing), adrenal insufficiency (impaired processing of proCRH), hyperghrelinemia (impaired proghrelin processing), and hypothyroidism (impaired proTRH processing). Individuals with inactivating mutations in PCSK1 phenocopy PWS patients in many aspects of the disease phenotype (Supplemental Table 3). PCSK1 hypomorphic patients also present with severe, early-onset hyperphagic obesity (multiple prohormone candidates), hypogonadotropic hypogonadism (impaired proGnRH processing), growth deficiency associated with low levels of circulating GH (impaired proGHRH processing), hypoadrenalism (impaired processing of CRH), and hyperproinsulinemia associated with hypoglycemia which can progress to type 2 diabetes later in life (impaired proinsulin processing) (22). Circulating ghrelin levels have not been measured in individuals hypomorphic for PCSK1; however, mice null for Pcsk1 display increased transcript levels of preproghrelin in the stomach associated with impaired stomach proghrelin processing. We identified that Snord116 $6^{p-/ m+}$ mice also display increased transcript levels of preproghrelin in the stomach associated with impaired stomach proghrelin processing. These data suggest that circulating total ghrelin levels are also likely increased in Pcsk1-null mice and humans hypomorphic for PCSK1.
Based on the data presented in Figures 1-3 and the known consequences of PC1 deficiency, we hypothesize that PC1 deficiency in the central nervous system - due to paternal deletion of SNORD116 - is a major cause of hyperphagic obesity in PWS. However, the precise molecular physiology by which deficiency of PCSK1 drives hyperphagic obesity has not been definitively determined at present. While impaired processing of POMC to $\alpha$-MSH is an obvious candidate for the hyperphagia, POMC transcript and protein levels can be upregulated in the setting of PC1 deficiency and could compensate for impaired POMC processing (likely due to impairment of a negative feedback regulatory mechanism) $(23,46)$. There are conflicting reports in the literature regarding $\alpha$-MSH levels in mouse models of PC1 deficiency. Initial studies of PC1-deficient mice reported reductions in hypothalamic and pituitary $\alpha$-MSH by gel filtration and RIA; however, follow-up studies using liquid chromatography-mass spectrometry (LC-MS) did not detect reductions in pituitary $\alpha-\mathrm{MSH}(23,46,50)$. The quantitative LC-MS data suggest that other prohormones in the central nervous system that are processed by PC1 may account for the hyperphagia in PC1-deficient models.

In addition to POMC, many other hormones involved in food intake and energy expenditure are processed by PC1, including prohormones for AgRP, NPY, CART, oxytocin, and brain-derived neurotrophic factor (BDNF) (22). Hypothalamic levels of the orexigenic ProAgRP are increased in Pcsk1 hypomorphs (28). We found that hypothalami of Snord116 $6^{-/ m+}$ mice have increased transcript levels of Npy and Agrp and decreased transcript levels of Pcsk1. Processing of AgRP is not necessary for its biological activity, and ProAgRP has considerable antagonistic activity toward $\alpha-\mathrm{MSH}$ at melanocortin 4 receptor (MC4R) $(27,28)$. Thus, increased hypothalamic levels of ProAgRP could drive hyperphagia.

Oxytocin is an anorexigenic hormone important for the regulation of body weight; the pro-form of oxytocin is cleaved by PC1 (50). It is unclear whether pro-oxytocin is bioactive. Pituitary processed oxytocin levels are severely reduced in pituitaries of PC1-null mice (hypothalami were not assessed) (50). Oxytocin-producing neurons in the paraventricular nucleus (PVN) and supraoptic nucleus (SON) express MC4R, the receptor for $\alpha-\mathrm{MSH}$ (endogenous ligand) and AgRP (endogenous antagonist) (51). Central and peripheral administration of oxytocin reduces food intake in humans, rhesus macaques, rats, and mice (51-53). Oxytocin receptor -knockout mice weigh more $(16 \%)$ than WT littermates; however, the mechanism of increased body weight remains unclear (54). Oxytocin is produced in Sim1-expressing neurons in the PVN (55). Patients with inactivating mutations in SIM1 have severe, early-onset hyperphagic obesity (56). Mice that are haploinsufficient for Sim1 are hyperphagic and obese and have reductions in PVN oxytocin levels; administration of exogenous oxytocin reverses hyperphagia in $\mathrm{Siml}^{+/-}$mice (55). These data indicate that hypothalamic oxytocin likely contributes to the regulation of body weight directly downstream of melanocortin signaling. Thus, improper pro-oxytocin processing resulting in reduced levels of oxytocin in the setting of PC1 deficiency is a plausible candidate mechanism for the associated hyperphagia.

Swaab et al. identified a reduction in the number of oxytocin-positive neurons in the PVH of post mortem brains of PWS patients (57). Adult PWS patients have been reported to have 
decreased plasma oxytocin levels, while pediatric PWS patients (aged 5-11 years) have increased plasma oxytocin levels $(58,59)$. Phenotypes of PWS - including impaired suckling in infants and behavioral symptoms consistent with autism spectrum disordercould be due to impaired oxytocin function $(60,61)$.

$B D N F$ is highly expressed in the hypothalamic paraventricular and ventromedial nuclei; while NTRK2 (nominal mature $\mathrm{BDNF}$ receptor) is highly expressed in the paraventricular and ventromedial hypothalamic nuclei, as well as the lateral hypothalamic area. BDNF has been implicated in regulation of human body weight as a molecule able to increase energy expenditure and decrease food intake. In common obesity, as well as in PWS, serum BDNF levels are reduced (62). BDNF is also synthesized as a prohormone. ProBDNF can be cleaved by $\mathrm{PC7}$ and furin to mature BDNF, which is released by both the regulated and constitutive secretory pathways (63). Hypothalamic BDNF involved in the control of body weight is most likely secreted by the regulated secretory pathway. Pcsk $7^{-1}$ mice are not obese, whereas $B d n f^{4--}$ mice are obese (64). Pcsk1 expression is upregulated in injured nerve cells in association with an increased demand for proBDNF and proNGF cleavage. It been suggested that PC1 is capable of processing proBDNF (65). Although speculative, Stijnen and others have postulated that $\mathrm{PC} 1$ may process proBDNF in the regulated secretory pathway in neurons, such as in the PVH, involved in the regulation of food intake and energy expenditure (66). Hypothalamic or circulating levels of BDNF have not been assessed in models of PC1 deficiency. Further studies are needed to determine whether hypothalamic processing of pro-BDNF is impaired in Pcsk1-null mice or iPSC-derived neurons hypomorphic for PC1. ProBDNF processing should also be assessed in mouse and iPSC-derived neuron models of PWS.

Thus, the impaired processing of 3 candidate hormones besides POMC - pro-oxytocin, proBDNF, or proAgRP - may contribute to hyperphagia in the setting of PC1 deficiency, and thus potentially PWS. These possibilities are not mutually exclusive. Further studies are necessary to determine whether these hormones are misprocessed in the hypothalamic neurons of mice and humans (iPSCderived neurons) hypomorphic for PCSK1 or SNORD116.

Nhlh2 colocalizes with $33 \%$ of Pomc-expressing neurons in the rostral arcuate nucleus and $41 \%$ of Trh-expressing neurons in the PVN (17). In Nhlh2 $2^{--}$mice, the prohormone processing of POMC to $\alpha-\mathrm{MSH}$ and adrenocorticotropic hormone (ACTH) is impaired, as well as prohormone processing of proTRH to TRH (17). Nhlh2, PC1, and PC2 are physiologically regulated by nutritional status and leptin (20). Leptin can increase NHLH2, PCSK1, and PCSK2 promoter activity through STAT3-dependent mechanisms in mouse hypothalamus and 293T cells, respectively (20). For TRH, leptin can couple the upregulation of TRH transcription to increased proTRH processing (20). Individuals with PWS, and Snord $116^{p-/ m+}$ mice, produce appropriate quantities of leptin relative to fat mass; however, it is unclear whether the regulation of NHLH2, PCSK1, and PCSK2 by leptin is affected in PWS individuals or mouse models (Supplemental Figure 17).

Most prohormones, including proinsulin and POMC, are much less biologically active than their processed forms. Sertoli cells exposed to prepro-GHRH-(75-92)- $\mathrm{NH}_{2}$ increase cellular
cAMP levels, while Sertoli cell exposed to prepro-GHRH-(75-83) do not change cellular cAMP levels (67). However, mice null for PC1 produce increased levels of proGHRH and decreased levels of GHRH compared with WT littermates and are very clearly runted (46). Thus, it seems unlikely that proGHRH retains physiologically significant agonist activity at GHRH receptor (GHRHR). Short-term exogenous administration of recombinant rat proghrelin to mice increases food intake and energy expenditure, resulting in a net body weight loss (68). Treatment of HEK-293 cells transfected with Ghsr-1a increases intracellular calcium signaling in response to synthetic ghrelin but not in response to synthetic proghrelin (68). The biological effects and mechanism of action of synthetic proghrelin appear to be distinct from those of mature ghrelin (68).

Snord116 ${ }^{p-/ m+}$ mice are runted and do not develop obesity. $P w_{c} r^{p-/ m+}$ mice, which carry a paternal deletion in a critical region of the PWS locus, segregate for a slightly different deletion of the Snord116 gene cluster. These mice are also runted and do not develop obesity. Knockin of a 5'HPRT-LoxP-Neo ${ }^{\mathrm{R}}$ cassette 27 $\mathrm{kb}$ upstream of Snord116 on the maternal allele induces maternal expression of Snord116. PWScr $r^{-/ m 5^{\prime} L o x P}$ mice, which have a 5'HPRT-LoxP-NeoR cassette (5'LoxP) inserted upstream of the maternal PWScr allele, are not runted and have body weights indistinguishable from those of WT mice. Pcsk1-null mice are also runted and do not become obese. The growth curves for the Pcsk1-null and Snord116 $6^{p-/ m+}$ mice are very similar. However, the Pcsk1 $1^{\text {N222D }}$ mouse (hypomorphic for PC1), is obese and not runted. Furthermore, $\mathrm{Pcsk1}^{+-}$mice have increased body weights compared with WT littermates and likewise are not runted. These data suggest that there may be a critical threshold for PC1 levels important to growth and adiposity.

The human PWS microdeletion patient cells utilized in this study harbor a paternal deletion of SNORD116, IPW, and SNORD109A. Without isogenic models of SNORD116, IPW, and SNORD109A deficiency, we cannot definitively conclude whether SNORD116, SNORD109, and IPW are, individually or in combination, the main drivers of downregulation of NHLH2 and PCSK1. Although the downregulation of Nhlh2 and Pcsk1 in Snord116 ${ }^{p-/ m+}$ mice suggests that paternal deletion of just Snord116 is at least sufficient for downregulation of Nhlh2 and Pcsk1 in vivo.

SNORD116 is an imprinted, paternally expressed, noncoding RNA cluster that contains thirty snoRNAs that are $85 \%$ homologous to one another, 5 sno-lnc RNAs, and one long noncoding RNA, 116HG. We have implicated NHLH2 and PCSK1 as major molecular targets of SNORD116, although it is unclear whether there are direct interactions between SNORD116 and PCSK1 and/ or SNORD116 and NHLH2. It is also unclear whether such interaction(s) were to occur, exactly which noncoding RNA sequences encoded from the SNORD116 snoRNA cluster would interact with PCSK1 and/or NHLH2. Furthermore, it is not known whether such interactions would occur at the RNA-DNA, RNA-RNA, or RNA-protein levels. It is also likely that there are targets of SNORD116 other than PCSK1 and NHLH2 that may be clinically relevant in PWS. It is possible that other targets of SNORD116 may account for aspects of the PWS phenotype that are not present in PC1-deficient patients, such as delayed gastric emptying, characteristic PWS facial features, and behavioral phenotypes such as skin picking. 


\section{Methods}

Human subjects. All studies and consenting procedures were approved by the Institutional Review Boards of the participating institutions including Columbia University Medical Center, University of Florida, and University of Kansas.

iPSC and PSC culture methods. Human iPSC cultures were generated and maintained as previously described (14).

Neuronal differentiation. Neuronal differentiation was performed as previously described (14).

FACS for CD56. Cells were enzymatically dissociated with Accutase and filtered through a $35-\mathrm{mm}$ cell strainer (BD Biosciences) to obtain a single-cell suspension prior to resuspension in $100 \mu \mathrm{l}$ of a sterile iPSC staining buffer (DPBS containing 0.5\% BSA fraction V [Invitrogen], 100 $\mathrm{U} / \mathrm{ml}$ penicillin/streptomycin [Invitrogen], $2 \mathrm{mM}$ EDTA [Invitrogen], and $20 \mathrm{mM}$ glucose [Sigma-Aldrich]). CD56-V450 (1 $\mu$ l; BD Biosciences, 560360) or Stem Cell Technologies Anti-Human CD56 (NCAM) antibody (60021AZ) was added to the cells and incubated at room temperature for 15 minutes shielded from light. The stained cells were washed once with iPSC staining buffer and sorted immediately on a 5 laser BD Biosciences ARIA-IIu SOU Cell Sorter configured with a $100-\mu \mathrm{m}$ ceramic nozzle and operating at 20-psi sheath fluid pressure. Cells were sorted into a 15-ml tube containing growth media as described above.

RNA sequencing. CD56 ${ }^{+}$iPSC-derived neurons were immediately pelleted following FACS. Total RNA was isolated from CD56 ${ }^{+}$iPSC-derived neurons using the Norgen Biotek Total RNA Purification Micro Kit with DNAse treatment. RNA sequencing was performed at the Columbia Genome Center, $1 \times 100$-bp read length, 30-M read count. RNA sequencing was performed on CD56 ${ }^{+}$iPSC-derived neurons from 7 unaffected control iPSC/ESC lines, 2 PWS LD iPSC lines, and 1 (2 clones were used) PWS microdeletion iPSC line. Reads were mapped to a reference genome (human: NCBI/build37.2; mouse: University of California Santa Cruz [UCSC] $/ \mathrm{mm} 9$ ) using Tophat software with 4 mismatches (--read-mismatches $=4)$ and 10 maximum multiple hits (--max-multihits $=10)$. The relative abundance (FPKM value) of transcripts was estimated using default settings in Cufflinks software (version 2.0.2; http://cole-trapnelllab.github.io/cufflinks/). Transcripts were then sorted in Microsoft Excel by FPKM status; genes with "low data" or "fail" FPKM status (189 genes) were not considered for analysis. Only FPKM values of "OK" were kept. FPKM OK status was determined by Cufflinks software to have adequate coverage. The average FPKM values were then calculated for each genotype: unaffected control (CON), PWS microdeletion (MD), and PWS LD (LD). The ratios of differential gene expression (DE) between PWS LD and unaffected control and PWS microdeletion and unaffected control were then calculated. DE ratios $>2$ and $<0.5$ were considered as upregulated or downregulated, respectively. A 2-tailed, type $3 t$ test was performed for all genes; $P$ values less than 0.05 were considered significant.

qRT-PCR on iPSC-derived neurons. RNA was isolated from PSCs using the QIAGEN RNeasy kit with DNAse treatment. RNA was isolated from iPSC-derived neurons using the Norgen Biotek Total RNA Purification Micro Kit with DNAse treatment. Total RNA was converted to cDNA using the Roche Transcriptor First Strand cDNA Synthesis Kit. qRT-PCR was performed using Roche LightCycler 480 SYBR Green I Master mix. Primers are listed in Supplemental Table 4. All primers used for a qRTPCR assays were validated using a 5-point standard curve. TBP was used as a housekeeping gene, and fold change was calculated using the $2^{-\Delta \Delta C t}$ method (Figure 1, B-F, H, and K; $n=7$ unaffected control PSCs, $n=1$ PWS microdeletion [ 2 clones used], $n=3$ PWS LD lines used).
Western blot in iPSC-derived neurons for NHLH2 and PC1. Protein levels of NHLH2 (WH0004808M1, Sigma-Aldrich), PC1 (11914 Cell Signaling Technology), and $\alpha$-tubulin (2144 or 3873 [clone DM1A], Cell Signaling Technology) were examined by Western blot analysis. Whole cell lysates from D34 PSC-derived neurons were obtained by reconstituting and lysing frozen cell pellets $\left(-80^{\circ} \mathrm{C}\right)$ in RIPA buffer (Thermo Fisher, 89900) with protease inhibitors (Halt protease and phosphatase inhibitor cocktail, Thermo Scientific, 78442). Samples were sonicated for 15 seconds. Total protein content was quantified using the Pierce BCA Protein assay kit (Thermo Fisher, 23227). Total protein $(15 \mu \mathrm{g})$ was mixed with sample reducing agent (NuPAGE, NP0009) and LDS sample buffer (NuPAGE, NP0008); samples were incubated at $90^{\circ} \mathrm{C}$ for 5 minutes. Total protein $(15 \mu \mathrm{g})$ was run on $4 \%-12 \%$ Bis-Tris mini gels (NuPAGE, NP0321) with MOPS SDS running buffer (Novex Life Technologies, B0001); 1× antioxidant (NuPAGE, NP0005) was added to the inner chamber. Electrophoresis was run at $70 \mathrm{~V}$ for 15 minutes and then $200 \mathrm{~V}$ until desired molecular weight separation was achieved. Protein was transferred to nitrocellulose blots using the Invitrogen iBlot system run at P1. Primary antibody was incubated overnight at $4^{\circ} \mathrm{C}$ with gentle rocking. Blots were washed 3 times for 5 minutes in TBST. LI-COR secondary antibodies (IRDye 800 CW goat anti-rabbit, 926-32211; IRDye 680 LT donkey anti-mouse, 926-68022; IRDye $800 \mathrm{CW}$ goat anti-mouse, 926-32210; IRDye 680LT donkey anti-rabbit, 926-68023) were used at 1:5,000 dilution and incubated at room temperature with gentle rocking for 1 hour. Blots were washed twice in TBST for 5 minutes, then twice in TBS for 5 minutes. Blots were then imaged using the Odyssey Classic imaging system (LI-COR Biotechnology). Data were analyzed using Image Studio Lite version 5.0 and GraphPad Prism 6.

Mouse breeding, genotyping, and anthropometric measurements. All animal work was carried out with approval of the IACUC of Columbia University Medical Center under protocol AC-AAAH1203. Snord116 $6^{p-m+}$ mice on a C57BL/6J background were ordered from the Jackson Laboratory (stock number 008149). A male Snord116 $6^{p-/ m+}$ mouse was mated to WT C57BL/6J females. Ovulation cycles of WT C57BL/6J females were synced by exposure to male mouse urine. Offspring were genotyped using methods published by Ding et al. (11). Only male mice were kept for study. Mice were weighed weekly (Figure 3G, $n=4-20$ mice/ time point) and body composition was measured by NMR monthly using a Bruker Minispec TD NMR. Mice were fed 21.56\% fat breeder chow for the entirety of the study (Purina Irradiated 5058 Picomouse Diet 20). Mouse nasal to anal length was measured at 1 and 6 months of age immediately following sacrifice (Figure $3 \mathrm{H}, n=6 \mathrm{WT}, n=7$ Snord$116^{p-m+}$ mice/time point).

Intraperitoneal glucose tolerance test in Snord116 deletion mice; ELISA for proinsulin, insulin, and C-peptide; islet isolation procedures; expression levels of PC1 and PC2. Mice were fasted overnight (16 hours). In the morning, mice were intraperitoneally injected with $50 \%$ dextrose at a dose of $3 \mathrm{mg}$ glucose per kg body weight (Hospira Inc., NDC 04096648702). Blood glucose was assayed at time 0 (fasting, prior to injection) and 30 minutes following injection using a FreeStyle Lite blood glucose meter and strips (accurate range 30-372 mg/dl) ( $n=9 \mathrm{WT}, n=7$ Snord116 $6^{p-m+}$ mice). Whole blood was also collected into heparin tubes on ice at time 0 and 30 minutes after injection. Heparin-treated blood was centrifuged at 2,000 RCF for 15 minutes at $4^{\circ} \mathrm{C}$; plasma was collected and frozen at $-80^{\circ} \mathrm{C}$ for analysis of insulin and proinsulin content. Proinsulin, insulin, and C-peptide content was measured by ELISA (Crystal 
Chem Ultra Sensitive Mouse Insulin ELISA Kit, 90080; Mercodia Rat/ Mouse Proinsulin ELISA, 10-1232-01; ALPCO Mouse C-peptide ELISA, 80-CPTMS-E01). Prior to islet isolation, mice were fasted overnight (16 hours). Mice were sacrificed by cervical dislocation. A 30-gauge needed connected to a $5-\mathrm{ml}$ syringe filled with $50 \% \mathrm{wt} /$ vol collagenase P (dissolved in M199 media with 1\% penicillin-streptomycin (Pen Strep) (Roche, 11249002001; Gibco, 31150022) was inserted into the common bile duct, and the pancreas was perfused with collagenase P. Islet isolation was carried out as described previously (69). After picking, islets recovered overnight ( 16 hours) in RMPI media (Thermo Fisher, 11875) with $15 \%$ FBS, $1 \%$ Pen Strep, $1 \%$ GlutaMAX at $37^{\circ} \mathrm{C}, 5 \% \mathrm{CO}_{2}$. The following day, islets were placed into 1.7-ml microcentrifuge tubes (40 islets/ tube for RNA analysis; $n=6 \mathrm{WT}, n=5$ Snord11 $6^{p-/ m+}$ mice; islets were pooled according to genotype, 20 islets/tube for protein analysis; $n=15$

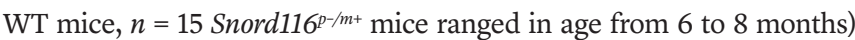
containing $200 \mu$ l of basal media, RMPI media (Thermo Fisher, 11879) with $1 \%$ Pen Strep, 1\% GlutaMAX, 2 mM glucose. Islets were allowed to recover for 1 hour. Islets were then incubated in basal media for 1 hour, high glucose (20 mM glucose) for 30 minutes (for RNA collection) or 90 minutes (for protein analysis). Total RNA was isolated as described for PSC-derived neurons. Primer sequences are listed in Supplemental Table 4. Protein levels of PC1 (Cell Signaling Technology, 11914 or Sigma-Aldrich, WH0005122M2 clone 3D2), PC2 (Cell Signaling Technology, 14013, clone D1E1S), and $\beta$-actin (Cell Signaling Technology, 3700) were probed as described for PSC-derived neurons.

qRT-PCR of liver Igfl. Livers from $\mathrm{p} 30$ ad libitum fed animals were homogenized in QIAzol, followed by phenol chloroform separation and precipitation with $70 \%$ ethanol of the aqueous fraction, which was subsequently applied to QIAGEN RNeasy columns. DNase treatment was performed. qRT-PCR was performed as described for PSC-derived neurons ( $n=6 \mathrm{WT}, n=7$ Snord $116^{p-m+} \mathrm{P} 30$ male mice). Bact was used as a housekeeping gene control for all mouse qRT-PCR assays, and fold change was calculated using the $2^{-\Delta \Delta \mathrm{Ct}}$ method.

qRT-PCR in hypothalamus of 5-hour-fasted/refed mice. Mice were fasted overnight (16 hours) and refed for 5 hours. Mice were individually housed in order to obtain food intake measurements on a per-mouse basis. One cohort of animals was sacrificed following an overnight (16 hours) fast. Another cohort was sacrificed following an overnight (16 hours) fast with 5 -hour refeeding. Mice were sacrificed by cervical dislocation. The hypothalamus was dissected out of the brain using a brain block and clean razor blades. Hypothalami were immediately homogenized in QIAzol, and subsequent isolation of RNA was carried out as described for liver. RT and qPCR were performed as described for PSC-derived neurons; primers are listed in Supplemental Table 4 ( $n=11 \mathrm{WT}, n=13 \mathrm{DEL}$ [Snord116 $\mathrm{p}-/ \mathrm{m}+$ ] adult males, overnight fasted; $n=15 \mathrm{WT}, n=14$ DEL adult males, 5-hour refeed).

IGF1 ELISA. Circulating IGF1 levels were measured from ad libitum fed 1-month-old mice; the Mouse/Rat IGF-I Quantikine ELISA Kit MG100 from R\&D Systems was used ( $n=6 \mathrm{WT}, n=7$ Snord116 $6^{p-/ m+}$ P30 male mice).

ProGHRH/GHRH measurements. Hypothalami were dissected out of 6-week-old overnight-fasted (16 hours) male mice using a brain block and clean razor blades (changed between each mouse). Preparation of hypothalamic extract was done according to Miki et al.; hypothalamus was immediately placed into $500 \mu \mathrm{l}$ of $1 \mathrm{~N}$ acetic acid, $0.02 \mathrm{~N} \mathrm{HCl}, 10$ $\mathrm{mM}$ EDTA, $1 \mu \mathrm{g} / \mathrm{ml}$ pepstatin (70). These extracts were then lyophilized and resuspended in $100 \mu \mathrm{l}$ of $3 \mathrm{~N}$ acetic acid. Further sample preparation and protein gel electrophoresis conditions were the same as for the stomach lysate Western blot probing for ghrelin. Electrophoresis was stopped once the desired molecular weight separation between mature GHRH, $5.2 \mathrm{kDa}$, and proGHRH, $12.3 \mathrm{kDa}$, was achieved, as visualized by the SeeBlue Plus2 Pre-Stained molecular weight standard (Life Technologies, LC5925). Clean razor blades were then used to excise gel slices from the proGHRH and mature GHRH fractions for each sample lane at $12.3 \mathrm{kDa}$ and $5.2 \mathrm{kDa}$, respectively. Gel slices were placed into $1.0 \mathrm{ml}$ of elution buffer (50 mM Tris-Hcl, 150 mM NaCl, 0.1 mM EDTA; pH 7.5) in clean 1.7-ml microcentrifuge tubes. Gel pieces were then chopped into much smaller pieces approximately of uniform size, to increase the surface area from which protein could passively diffuse into elution buffer with clean surgical scissors. The chopped gel slice and elution buffer mixture was then incubated on a shaking heat block overnight at $50^{\circ} \mathrm{C}$. The following day, samples were centrifuged at 10,000 $g$ for 15 minutes, and supernatant was collected. Immunoreactive GHRH content was then measured using a GHRH ELISA kit (Clone-Cloud Corp. ELISA Kit for Growth Hormone Releasing Hormone, CEA438Mu). Six-week-old male mice were used ( $n=14 \mathrm{WT}, n=11 \mathrm{DEL}$, error bars are SEM in Figure 3L).

Pituitary GH Western blotting. Pituitaries were removed from overnight-fasted (16 hours) 4-week-old male mice. De-brained skulls were placed in Petri dishes with PBS. While viewing pituitary in de-brained skull via dissecting microscope, fine-tip tweezers were used to excise pituitary out of skull. Use of the PBS-filled Petri dish prevented pituitaries from folding in on itself. Excised pituitaries were then flash frozen in $\mathrm{LN}_{2}$. Pituitaries were homogenized in RIPA buffer (Thermo Fisher, 89900) supplemented with protease inhibitors (Halt protease \& phosphatase inhibitor cocktail, Thermo Scientific, 78442). Five micrograms of total protein was loaded onto $4 \%-12 \%$ bis-tris gels, transferred to nitrocellulose membranes using iBlot program 0. Primary GH antibody (National Hormone and Peptide Program) in LI-COR blocking buffer and $1 \%$ Tween 20 was incubated for 2 hours at room temperature; blots were then washed 3 times for 5 minutes each in PBST. Blots were incubated with secondary antibodies at room temperature for 1 hour with gentle shaking. Blots were again washed 3 times for 5 minutes each in PBST. Two final washes were performed in PBS. Blots were imaged using the Odyssey Classic imaging system (LI-COR Biotechnology). Data were analyzed using Image Studio Lite version 5.0 and GraphPad Prism 6 ( $n=5$ WT, $n=4$ Snord116 $6^{p-m+} \mathrm{P} 30$ male mice).

Total ghrelin ELISA. Total circulating ghrelin was measured from adult male mice that were fasted overnight (16 hours). Whole blood was collected in the morning into heparinized tubes on ice, which was spun at 2,000 RCF at $4^{\circ} \mathrm{C}$ for 15 minutes. Total ghrelin content of plasma was measured using the Millipore Rat/Mouse Total Ghrelin ELISA kit EZRGRT-91K ( $n=21 \mathrm{WT}, n=17$ DEL adult males, fasted).

qRT-PCR for Snord116 and Ghrl, and Western blot for PC1 in P3O stomachs. Whole stomachs were excised from overnight (16 hours) fasted 1-month-old (P30) male mice. Stomachs were cut open sagitally, and residual food content was removed from stomachs. Stomach tissue was rinsed twice in room-temperature PBS before flash freezing in liquid nitrogen. Frozen stomachs were then cryohomogenized (Cryo-Cup Grinders, Research Products International, catalog 206), and 25\% of the homogenate was used for RNA analysis, while $75 \%$ was used for protein analysis. RNA was isolated as described for liver. For protein analysis the homogenate was further homogenized briefly in RIPA buffer (Thermo Fisher, 89900) supplemented with protease inhibitors (Halt protease \& phosphatase inhibitor cocktail, Thermo Scientific, 
78442) using a handheld homogenizer. Lysates were centrifuged at for 20 minutes at 20,000 RCF at $4^{\circ} \mathrm{C}$; clear supernatant was collected for analysis. Western blot for PC1 was performed as described for iPSC-derived neurons ( $n=6 \mathrm{WT}, n=6 \mathrm{DEL}$ P30 male mice).

Western blot for proghrelin/ghrelin in stomachs. Stomach lysates from adult mice fasted overnight (16 hours) were probed for ghrelin content by Western blot following the methods based on that of Zhu et al. and Yang et al. $(40,71)$. Stomachs were dissected out from the mouse and rinsed

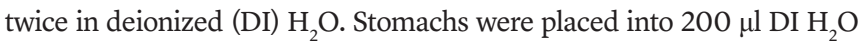
and boiled for 10 minutes using a water bath. Boiled stomachs were then cooled on ice. Next, stomachs were minced, and $200 \mu \mathrm{l} 2 \mathrm{M}$ acetic acid, $0.04 \mathrm{~N} \mathrm{HCl}$ were added such that the final concentration was $1 \mathrm{M}$ acetic acid and $0.02 \mathrm{~N} \mathrm{HCl}$. This mixture was then homogenized. Stomach homogenate was centrifuged at $20,000 \mathrm{~g}$ for 1.5 hours at $4^{\circ} \mathrm{C}$ in order to obtain a clear supernatant. Supernatant was collected and reduced to 300 $\mu \mathrm{l}$ in a vacuum centrifuge. Acetone precipitation was then performed. Acetone was cooled to $-20^{\circ} \mathrm{C} .600 \mu \mathrm{l}(2 \times$ volume of sample) acetone was added to the protein sample. Samples were incubated overnight at $-20^{\circ} \mathrm{C}$. Samples were then centrifuged for 10 minutes at 15,000 RCF at $4^{\circ} \mathrm{C}$. Supernatant was collected because ghrelin is dissolved in the hydrophobic fraction. The supernatant was then dried in vacuo overnight. Dried protein was then resuspended in $30 \mu \mathrm{DI} \mathrm{H} \mathrm{H}_{2} \mathrm{O}$. Total protein content for each sample was determined by BCA assay. Ten micrograms total protein was mixed with loading buffer such that final concentrations were $0.1 \mathrm{M}$ Tris-chloride at pH 6.8, 5\% wt/vol SDS, $0.1 \mathrm{M}$ DTT, $5 \%$ glycerol. Sample loading buffer mixture was then incubated at $100^{\circ} \mathrm{C}$ for 5 minutes. Ten micrograms total protein was loaded onto a $16 \%$ tricine gel which was run in Tricine-SDS running buffer. $500 \mu \mathrm{l}$ antioxidant was added to the center chamber. The gel was run at $70 \mathrm{~V}$ for 15 minutes, then $126 \mathrm{~V}$ until optimal migration was achieved. Proteins were transferred from the electrophoresis gel to a PVDF membrane using the iBlot system. Transfer conditions were $20 \mathrm{~V}$ for 5 minutes (program 3 run for 5 minutes). PVDF blots were immediately placed in PBST. To prevent diffusion of the 3.4-kDa mature ghrelin, blots were fixed at room temperature for 15 minutes (on a shaker) in 50 mM HEPES-NaOH, pH 7.4, and containing 2.5\% wt/vol glutaraldehyde. The membrane was then washed 3 times, 5 minutes each, in PBST. The membrane was blocked for 30 minutes at room temperature in LI-COR blocking buffer with $1 \%$ Tween 20 . The blot was exposed to antighrelin (Santa Cruz Biotechnology Inc., sc-10368) and anti-preproghrelin (Pheonix Pharmaceuticals, H-031-34) primary antibodies during an overnight incubation at $4^{\circ} \mathrm{C}$ and gentle shaking. Blots were then washed 3 times for 5 minutes each in PBST. Blots were incubated with secondary antibodies at room temperature for 1 hour with gentle shaking. Blots were again washed 3 times for 5 minutes each in PBST. Two final washes were performed in PBS. Blots were imaged using the Odyssey Classic imaging system (LI-COR Biotechnology). Data were analyzed using Image Studio Lite Version 5.0 and GraphPad Prism 6 ( $n=9$ WT; $n=7$ Snord116 deletion; $n=2$ PC1-null adult mice fasted overnight).

Measurement of proinsulin, insulin, and glucose in human plasma. Plasma glucose levels were measured using the Wako Diagnostics AUTOKIT GLUCOSE C2. Proinsulin was measured using a Human Total Proinsulin ELISA kit (Millipore, EZHPI-15K). Insulin was measured using an Ultrasensitive Human Insulin ELISA kit (Mercodia, 10-1132-01) ( $n=25$ age/BMI-matched controls, $n=16$ PWS patients, $n=1$ PCSK1 mutation patient). All patient plasma collected at fasting. Unaffected control/patient genotype, sex, age, BMI $Z$ score, and weight are listed in Supplemental Table 2.
Neuron IHC. iPSC-derived neurons were stained with the neural makers: TUJ1 (Sigma-Aldrich, T2200), NCAM (Santa Cruz Biotechnology Inc., sc-106) and Ki-67 (Abcam, ab15580). The staining procedure was the same as for iPSCs.

Calorimetry of Snord116 deletion mice and WT littermates. Food intake and energy expenditure were monitored using a LabMaster CaloSys calorimetry system (TSE Systems). The first 24 hours was an acclimation period; data from this period were not used in analyses. Mice were weighed, and body composition was measured using the Bruker Minispec TD NMR prior to and following housing in the calorimetry system. Mice were maintained on breeder chow while in the calorimetry system ( $n=10 \mathrm{WT}, n=9$ deletion, 6-month-old males).

Leptin ELISA. The R\&D Systems Mouse/Rat Leptin Quantikine ELISA Kit (SMOBO0) was used to measure leptin from ad libitum fed male mice at 1 month of age and 6 months of age ( $n=13 \mathrm{WT}, n=13$ deletion).

Accession numbers. The genomic data reported here were deposited in the NCBI's Gene Expression Omnibus (GEO) database (GEO GSE89991).

Statistics. Data are presented as mean \pm SEM. Comparisons between 2 groups were analyzed using 2-tailed Student's $t$ tests. Comparisons among more than 2 groups were analyzed with 1-way ANOVA with the post hoc Tukey test, except for data in Figure 1, B-F, which were analyzed with Kruskal-Wallis and the post hoc Dunn's test. $P \alpha$ values less than 0.05 were considered significant. Asterisks throughout indicate ${ }^{*} P<0.05,{ }^{* *} P<0.01,{ }^{* *} P<0.001,{ }^{* * *} P<0.0001$.

Study approval. The studies using human plasma or human iPSC-derived neurons in this work were approved by the Columbia University Medical Center Institutional Review Board, Human Research Protection Office, New York, New York, USA. All subjects provided written informed consent prior to their participation in the study. The studies using mice in this work were approved by the IACUC of Columbia University Animal Care Facility.

\section{Author contributions}

LCB, CAL, DE, and RLL designed experiments. LCB, CAL, CRS, DP, RR, MZ, JFMC, MVM, AAS, GH, BL, and DE performed experiments. SE, JPS, and MT provided PWS microdeletion fibroblast line 066, PWS LD fibroblast line 031, and one unaffected control fibroblast line, 056. CRS and DJD provided PWS LD fibroblast lines 129 and 139, as well as plasma from individuals with PWS and unaffected controls. MGB, KC, BD, CP, MR, and IF provided human plasma samples. RD provided tissues from PC1-null mice. LW provided technical expertise. LCB, CAL, DJD, BL, DE, and RLL analyzed and interpreted data. LCB and RLL wrote the manuscript.

\section{Acknowledgments}

We thank Kylie Foo, Giselle Dominguez Gutierrez, and Lori Sussel, Columbia University for valuable instruction and advice on islet isolation techniques; Deborah J. Good, Virginia Tech, for supplying Nhlh2 primer sequences; Tiffany Thomas and Henry Ginsburg for lyophilization of hypothalamic tissue; Roxane Desjardins and Sandra Gagnon of Sherbooke University for tissues from PC1-null animals; Fred Kweh, University of Florida, for sending oral glucose tolerance test (OGTT) plasma; Bryan Wharram and Roy Smith for access to their ghrelin antibody; Sharon Wardlaw, Lori Zeltser, Utpal Pajvani, Claudia A. Doege, 
Yiying Zhang, Elizabeth Millings, Haiqing Hua, Linshan Shang, Bjarki Johanessen, Stuart G. Fisher, Andrew Sproul, Samson Jacob, Shaohua Xu, Scott Noggle, and Steven Chang for valuable discussions. We thank the following foundations/funding agencies for their support of this project: Foundation for Prader-Willi Research (FPWR) grants, FPWR/PWSA (Best Idea Grant), Rudin Foundation, New York Stem Cell Foundation (NYSCF), Helms- ley Foundation, Russell Berrie Foundation, NIH (R01DK52431; P30 DK26687: the New York Obesity Research Center).

Address correspondence to: Lisa C. Burnett or Rudolph L. Leibel, 1150 St. Nicholas Avenue Russ Berrie Pavilion Room 620 New York, NY 10033; Phone: 212851 5315; E-mail: lmc2200@cumc.columbia.edu (L.C. Burnett); rl2332@cumc.columbia.edu (R.L. Leibel).
1. Smith A, et al. Birth prevalence of Prader-Willi syndrome in Australia. Arch Dis Child. 2003;88(3):263-264.

2. Angulo MA, Butler MG, Cataletto ME. Prader-Willi syndrome: a review of clinical, genetic, and endocrine findings. J Endocrinol Invest. 2015;38(12):1249-1263.

3. Cassidy SB, Schwartz S, Miller JL, Driscoll DJ. Prader-Willi syndrome. Genet Med. 2012;14(1):10-26.

4. Butler MG, Lee PDK, Whitman BY, eds. Management of Prader-Willi Syndrome. New York: Springer Science Business Media, Inc; 2006.

5. Sahoo T, et al. Prader-Willi phenotype caused by paternal deficiency for the HBII-85 C/D box small nucleolar RNA cluster. Nat Genet. 2008;40(6):719-721.

6. de Smith AJ, et al. A deletion of the HBII-85 class of small nucleolar RNAs (snoRNAs) is associated with hyperphagia, obesity and hypogonadism. Hum Mol Genet. 2009;18(17):3257-3265.

7. Duker AL, et al. Paternally inherited microdeletion at $15 \mathrm{q} 11.2$ confirms a significant role for the SNORD116 C/D box snoRNA cluster in Prader-Willi syndrome. Eur J Hum Genet. 2010;18(11):1196-1201.

8. Bieth $\mathrm{E}$, et al. Highly restricted deletion of the SNORD116 region is implicated in Prader-Willi Syndrome. Eur J Hum Genet. 2015;23(2):252-255.

9. Butler MG, Christian SL, Kubota T, Ledbetter DH. A 5-year-old white girl with Prader-Willi syndrome and a submicroscopic deletion of chromosome 15q11q13. Am JMed Genet. 1996;65(2):137-141.

10. Bervini S, Herzog H. Mouse models of PraderWilli Syndrome: a systematic review. Front Neuroendocrinol. 2013;34(2):107-119.

11. Ding F, et al. SnoRNA Snord116 (Pwcr1/MBII-85) deletion causes growth deficiency and hyperphagia in mice. PLoS One. 2008;3(3):e1709.

12. Skryabin BV, et al. Deletion of the MBII-85 snoRNA gene cluster in mice results in postnatal growth retardation. PLoS Genet. 2007;3(12):e235.

13. Bazeley PS, et al. snoTARGET shows that human orphan snoRNA targets locate close to alternative splice junctions. Gene. 2008;408(1-2):172-179.

14. Burnett LC, et al. Induced pluripotent stem cells (iPSC) created from skin fibroblasts of patients with Prader-Willi syndrome (PWS) retain the molecular signature of PWS. Stem Cell Res. 2016;17(3):526-530.

15. Chambers SM, Fasano CA, Papapetrou EP, Tomishima M, Sadelain M, Studer L. Highly efficient neural conversion of human ES and iPS cells by dual inhibition of SMAD signaling. Nat Biotechnol. 2009;27(3):275-280.

16. Coyle CA, Jing E, Hosmer T, Powers JB, Wade G, Good DJ. Reduced voluntary activity precedes adult-onset obesity in Nhlh2 knockout mice. Physiol Behav. 2002;77(2-3):387-402.

17. Jing E, Nillni EA, Sanchez VC, Stuart RC, Good DJ. Deletion of the Nhlh2 transcription factor decreases the levels of the anorexigenic peptides alpha melanocyte-stimulating hormone and thyrotropin-releasing hormone and implicates prohormone convertases I and II in obesity. Endocrinology. 2004;145(4):1503-1513.

18. Bittel DC, Kibiryeva N, Sell SM, Strong TV, Butler MG. Whole genome microarray analysis of gene expression in Prader-Willi syndrome. Am J Med Genet A. 2007;143A(5):430-442.

19. Fox DL, Good DJ. Nescient helix-loop-helix 2 interacts with signal transducer and activator of transcription 3 to regulate transcription of prohormone convertase 1/3. Mol Endocrinol. 2008;22(6):1438-1448.

20. Nillni EA. Regulation of prohormone convertases in hypothalamic neurons: implications for prothyrotropin-releasing hormone and proopiomelanocortin. Endocrinology. 2007;148(9):4191-4200.

21. Seidah NG. The proprotein convertases, 20 years later. Methods Mol Biol. 2011;768:23-57.

22. Stijnen P, Ramos-Molina B, O'Rahilly S, Creemers JW. PCSK1 mutations and human endocrinopathies: from obesity to gastrointestinal disorders. Endocr Rev. 2016;37(4):347-371.

23. Lloyd DJ, Bohan S, Gekakis N. Obesity, hyperphagia and increased metabolic efficiency in Pc1 mutant mice. Hum Mol Genet. 2006;15(11):1884-1893.

24. Yeo GS, Lank EJ, Farooqi IS, Keogh J, Challis BG, O'Rahilly S. Mutations in the human melanocortin-4 receptor gene associated with severe familial obesity disrupts receptor function through multiple molecular mechanisms. Hum Mol Genet 2003;12(5):561-574.

25. Mendiratta MS, et al. Early onset obesity and adrenal insufficiency associated with a homozygous POMC mutation. Int J Pediatr Endocrinol. 2011;2011(1):5.

26. Cyr NE, Stuart RC, Zhu X, Steiner DF, Nillni EA. Biosynthesis of proTRH-derived peptides in prohormone convertase 1 and 2 knockout mice. Peptides. 2012;35(1):42-48.

27. Wardlaw SL. Hypothalamic proopiomelanocortin processing and the regulation of energy balance. Eur J Pharmacol. 2011;660(1):213-219.

28. Creemers JW, et al. Agouti-related protein is posttranslationally cleaved by proprotein convertase 1 to generate agouti-related protein (AGRP) 83132: interaction between AGRP83-132 and melanocortin receptors cannot be influenced by syndecan-3. Endocrinology. 2006;147(4):1621-1631.

29. Zhu X, Orci L, Carroll R, Norrbom C, Ravazzola $\mathrm{M}$, Steiner DF. Severe block in processing of proinsulin to insulin accompanied by elevation of des-64,65 proinsulin intermediates in islets of mice lacking prohormone convertase $1 / 3$. Proc Natl Acad Sci U S A. 2002;99(16):10299-10304.

30. Stephens JW, et al. Comment on: Polonsky et al. Structured self-monitoring of blood glucose significantly reduces $\mathrm{A} 1 \mathrm{C}$ levels in poorly controlled, noninsulin-treated type 2 diabetes: results from the structured testing program study. Diabetes Care 2011;34:262-267. Diabetes Care. 2011;34(5):e57; author reply e58.

31. Jackson RS, et al. Small-intestinal dysfunction accompanies the complex endocrinopathy of human proprotein convertase 1 deficiency. J Clin Invest. 2003;112(10):1550-1560.

32. Kojima M, Hosoda H, Matsuo H, Kangawa K. Ghrelin: discovery of the natural endogenous ligand for the growth hormone secretagogue receptor. Trends Endocrinol Metab. 2001;12(3):118-122.

33. Feigerlová E, et al. Hyperghrelinemia precedes obesity in Prader-Willi syndrome. JClin Endocrinol Metab. 2008;93(7):2800-2805.

34. Kweh FA, et al. Hyperghrelinemia in Prader-Willi syndrome begins in early infancy long before the onset of hyperphagia. Am JMed Genet A. 2015;167A(1):69-79.

35. Haqq AM, et al. Serum ghrelin levels are inversely correlated with body mass index, age, and insulin concentrations in normal children and are markedly increased in Prader-Willi syndrome. JClin Endocrinol Metab. 2003;88(1):174-178.

36. Kuppens RJ, et al. Elevated ratio of acylated to unacylated ghrelin in children and young adults with Prader-Willi syndrome. Endocrine. 2015;50(3):633-642.

37. Butler MG, Bittel DC. Plasma obestatin and ghrelin levels in subjects with Prader-Willi syndrome. Am JMed Genet A. 2007;143A(5):415-421.

38. Butler MG, Bittel DC, Talebizadeh Z. Plasma peptide YY and ghrelin levels in infants and children with Prader-Willi syndrome. JPediatr Endocrinol Metab. 2004;17(9):1177-1184.

39. Cummings DE, et al. Elevated plasma ghrelin levels in Prader Willi syndrome. Nat Med. 2002;8(7):643-644.

40. Zhu X, Cao Y, Voogd K, Voodg K, Steiner DF. On the processing of proghrelin to ghrelin. J Biol Chem. 2006;281(50):38867-38870.

41. Tschöp M, Weyer C, Tataranni PA, Devanarayan V, Ravussin E, Heiman ML. Circulating ghrelin levels are decreased in human obesity. Diabetes. 2001;50(4):707-709.

42. Haqq AM, et al. Circulating ghrelin levels are suppressed by meals and octreotide therapy in children with Prader-Willi syndrome. J Clin Endocrinol Metab. 2003;88(8):3573-3576.

43. Goldstone AP, et al. Elevated fasting plasma ghrelin in prader-willi syndrome adults is not solely explained by their reduced visceral adi- 
posity and insulin resistance. JClin Endocrinol Metab. 2004;89(4):1718-1726.

44. DelParigi A, et al. High circulating ghrelin: a potential cause for hyperphagia and obesity in prader-willi syndrome. JClin Endocrinol Metab. 2002;87(12):5461-5464.

45. Posner SF, Vaslet CA, Jurofcik M, Lee A, Seidah NG, Nillni EA. Stepwise posttranslational processing of progrowth hormone-releasing hormone (proGHRH) polypeptide by furin and PC1. Endocrine. 2004;23(2-3):199-213.

46. Zhu X, et al. Disruption of PC1/3 expression in mice causes dwarfism and multiple neuroendocrine peptide processing defects. Proc Natl Acad Sci U S A. 2002;99(16):10293-10298.

47. Martín MG, et al. Congenital proprotein convertase $1 / 3$ deficiency causes malabsorptive diarrhea and other endocrinopathies in a pediatric cohort. Gastroenterology. 2013;145(1):138-148.

48. Ding F, et al. SnoRNA Snord116 (Pwcr1/MBII-85) deletion causes growth deficiency and hyperphagia in mice. PLoS One. 2008;3(3):e1709.

49. Marostica E, et al. The GHRH + arginine stimulated pituitary $\mathrm{GH}$ secretion in children and adults with Prader-Willi syndrome shows age- and BMI-dependent and genotype-related differences. Growth Horm IGF Res. 2013;23(6):261-266.

50. Pan H, et al. Neuropeptide processing profile in mice lacking prohormone convertase-1. Biochemistry. 2005;44(12):4939-4948.

51. Blevins JE, Ho JM. Role of oxytocin signaling in the regulation of body weight. Rev Endocr Metab Disord. 2013;14(4):311-329.

52. Blevins JE, et al. Chronic oxytocin administration inhibits food intake, increases energy expenditure, and produces weight loss in fructose-fed obese rhesus monkeys. Am J Physiol Regul Integr Comp Physiol. 2015;308(5):R431-R438.

53. Ott V, et al. Oxytocin reduces reward-driv- en food intake in humans. Diabetes. 2013;62(10):3418-3425.

54. Takayanagi Y, Kasahara Y, Onaka T, Takahashi N, Kawada T, Nishimori K. Oxytocin receptor-deficient mice developed late-onset obesity. Neuroreport. 2008;19(9):951-955.

55. Kublaoui BM, Gemelli T, Tolson KP, Wang Y, Zinn AR. Oxytocin deficiency mediates hyperphagic obesity of Sim1 haploinsufficient mice. Mol Endocrinol. 2008;22(7):1723-1734.

56. Bonnefond A, et al. Loss-of-function mutations in SIM1 contribute to obesity and Prader-Willi-like features. J Clin Invest. 2013;123(7):3037-3041.

57. Swaab DF. Prader-Willi syndrome and the hypothalamus. Acta Paediatr Suppl. 1997;423:50-54.

58. Höybye C, Barkeling B, Espelund U, Petersson M, Thorén M. Peptides associated with hyperphagia in adults with Prader-Willi syndrome before and during GH treatment. Growth Horm IGF Res. 2003;13(6):322-327.

59. Johnson L, Manzardo AM, Miller JL, Driscoll DJ, Butler MG. Elevated plasma oxytocin levels in children with Prader-Willi syndrome compared with healthy unrelated siblings. Am J Med Genet A. 2016;170(3):594-601.

60. Bennett JA, Germani T, Haqq AM, Zwaigenbaum L. Autism spectrum disorder in Prader-Willi syndrome: a systematic review. Am J Med Genet A. 2015;167A(12):2936-2944.

61. Yatawara CJ, Einfeld SL, Hickie IB, Davenport TA, Guastella AJ. The effect of oxytocin nasal spray on social interaction deficits observed in young children with autism: a randomized clinical crossover trial. Mol Psychiatry. 2016;21(9):1225-1231

62. Han JC, Muehlbauer MJ, Cui HN, Newgard CB, Haqq AM. Lower brain-derived neurotrophic factor in patients with Prader-Willi syndrome compared to obese and lean control subjects.
J Clin Endocrinol Metab. 2010;95(7):3532-3536.

63. Cunha C, Brambilla R, Thomas KL. A simple role for BDNF in learning and memory? Front Mol Neurosci. 2010;3:1.

64. Wetsel WC, et al. Disruption of the expression of the proprotein convertase $\mathrm{PC} 7$ reduces BDNF production and affects learning and memory in mice. Proc Natl Acad Sci U S A. 2013;110(43):17362-17367.

65. Marcinkiewicz M, Savaria D, Marcinkiewicz J. The pro-protein convertase $\mathrm{PC} 1$ is induced in the transected sciatic nerve and is present in cultured Schwann cells: comparison with PC5, furin and $\mathrm{PC7}$, implication in pro-BDNF processing. Brain Res Mol Brain Res. 1998;59(2):229-246.

66. Stijnen P, Tuand K, Varga TV, Franks PW, Aertgeerts B, Creemers JW. The association of common variants in PCSK1 with obesity: a HuGE review and meta-analysis. Am JEpidemiol. 2014;180(11):1051-1065.

67. Nillni EA, Steinmetz R, Pescovitz OH. Posttranslational processing of progrowth hormone-releasing hormone. Endocrinology. 1999;140(12):5817-5827.

68. Zhang W, Majumder A, Wu X, Mulholland MW. Regulation of food intake and body weight by recombinant proghrelin. Am J Physiol Endocrinol Metab. 2009;297(6):E1269-E1275.

69. Zmuda EJ, Powell CA, Hai T. A method for murine islet isolation subcapsular kidney transplantation.J Vis Exp. 2011;(50):e2096.

70. Miki N, et al. Characterization and localization of mouse hypothalamic growth hormone-releasing factor and effect of gold thioglucose-induced hypothalamic lesions. JNeuroendocrinol. 1994;6(1):71-78.

71. Yang J, Brown MS, Liang G, Grishin NV, Goldstein JL. Identification of the acyltransferase that octanoylates ghrelin, an appetite-stimulating peptide hormone. Cell.2008;132(3):387-396. 